\title{
Porosity of fluid-saturated porous media from measured seismic wave velocities
}

\author{
S. FOTI, ${ }^{*}$ C. G. LAI $\dagger$ and R. LANCELlOTTA
}

\begin{abstract}
This paper presents a novel procedure for determining porosity in fluid-saturated porous media from measured shear and dilatational wave velocities. The method is developed using the theory of linear poroelasticity in the low-frequency limit, which is equivalent to considering the fluid-saturated porous medium as a closed (undrained) system with the pore fluid moving in phase with the soil skeleton. The new procedure for the in-situ measurement of porosity has been applied at two sites in Italy using cross-hole seismic data. The results obtained are in excellent agreement with independent laboratory data gathered from undisturbed soil specimens.
\end{abstract}

KEYWORDS: dynamics; elasticity; in-situ testing; theoretical analysis
Dans cet article, les auteurs présentent un procédé novateur pour déterminer la porosité des matières poreuses saturées de liquide d'après les mesures de vélocité des ondes de cisaillement et de dilatation. Les auteurs développement cette méthode en utilisant la théorie de poroélasticité linéaire dans la limite de basse fréquence, ce qui revient à considérer la matière poreuse saturée de liquide comme système clos (non drainé), le liquide de pore se déplaçant en phase avec les particules du squelette. Les auteurs ont employé cette nouvelle méthode pour mesurer la porosité in situ sur deux sites en Italie en utilisant des données sismiques de forage transversal. Les résultats obtenus correspondent parfaitement aux données de laboratoires indépendantes obtenues à partir de spécimens de sol non perturbés.

\section{INTRODUCTION}

One of the main uses of seismic prospecting in geophysics and geotechnical engineering is for determining the fields of shear and dilatational wave velocities $V_{\mathrm{S}}$ and $V_{\mathrm{P}}$. These fields are important because they can be immediately related to the corresponding fields of stiffness and mechanical impedance, which serve as a basis for the geomechanical characterisation of a site. Since the seismic techniques used to measure $V_{\mathrm{S}}$ and $V_{\mathrm{P}}$ are based on the application of a small perturbation to the natural state of stress (represented by the geostatic stress tensor), they are ideal for determining the above parameters of mechanical behaviour, referring them to their initial, natural state.

The relationship between the measured quantities $V_{\mathrm{S}}$ and $V_{\mathrm{P}}$ and the corresponding parameters of mechanical behaviour is not unique, for it is inherently dependent on the choice of the constitutive model used to describe the mechanical response of geomaterials to perturbations of the stress field. Linear elasticity is the most frequently chosen constitutive assumption, and in this case $V_{\mathrm{S}}$ and $V_{\mathrm{P}}$ are directly related via the mass density of the medium to the parameters of mechanical behaviour, which in this case are the elastic shear and constrained moduli. However, by choosing a more refined constitutive model the quantities $V_{\mathrm{S}}$ and $V_{\mathrm{P}}$ can be related to other types of physical and constitutive parameter, which can then be determined through a process of inversion of the measured $V_{\mathrm{S}}$ and $V_{\mathrm{P}}$.

In the work presented in this paper, the theory of linear poroelasticity is used for determining porosity in fluidsaturated soil deposits from values of $V_{\mathrm{S}}$ and $V_{\mathrm{P}}$ measured

Manuscript received 27 September 2001; revised manuscript accepted 7 March 2002.

Discussion on this paper closes 1 December 2002, for further details see p. ii.

* Department of Structural and Geotechnical Engineering, Politecnico di Torino, Italy; currently Centre for Offshore Foundation Systems, University of Western Australia, Perth.

$\uparrow$ Studio Geotecnico Italiano, Milano, Italy.

\$ Department of Structural and Geotechnical Engineering, Politecnico di Torino, Italy. with cross-hole seismic tests. Porosity is an essential parameter for the description of the natural state of a soil deposit. Furthermore, experimental evidence shows that porosity strongly affects the mechanical, hydraulic and electric behaviour of geomaterials.

In fine-textured soils such as silts and clays, porosity is routinely measured in the laboratory on undisturbed specimens. However, in coarse-textured soils such as sands and gravels undisturbed sampling is difficult, since it requires the use of sophisticated freezing techniques (Hofman et al., 2000). With regard to empirical methods, an estimate of porosity could in principle be obtained from the initial shear modulus, $G_{\max }$, using correlations that relate this parameter to the void ratio and the effective confining pressure (Kramer, 1996). However, these methods suffer from an intrinsic limitation due to the dependence of these empirical correlations on soil type, mineralogy and stress history. Also, the procedures used in coarse-grained soil deposits based on the estimate (from penetration tests) of the relative density have certain limitations, since they are affected by an uncertainty that is difficult to quantify, and they require independent measurements of $e_{\min }$ and $e_{\max }$.

The theory of linear poroelasticity, the basic concepts of which were laid down by Biot (1956a; 1956b), predicts the existence in fluid-saturated media of two dilatational waves (dilatational waves of the first and second kind) and one rotational or shear wave that are dispersive: that is, their speed of propagation is frequency-dependent (Coussy, 1995). However, considering (a) the frequency content of the stress waves radiated by the sources typically used in seismic prospecting and (b) the values of permeability characteristic of most soils, it is easy to show that both the shear and the dilatational wave of the first kind propagate essentially in undrained conditions with no relative movement between the fluid and the solid phase. All these phenomena have already been predicted theoretically by using the theory of linear poroelastodynamics (Bourbie et al., 1987; Lancellotta, 2001; Miura et al., 2001).

The procedure used in this paper for computing porosity from measured values of $V_{\mathrm{S}}$ and $V_{\mathrm{P}}$ is based on the application of the theory of linear poroelasticity in the low 
frequency range. It is shown that the values of porosity obtained from the application of the proposed method at two sites in Italy are in very good agreement with independent results obtained from laboratory tests carried out on undisturbed soil specimens.

\section{WAVE PROPAGATION IN FLUID-SATURATED \\ POROELASTIC CONTINUA}

The equations of motion governing the propagation of mechanical disturbances in a deformable, fluid-saturated porous medium were first presented by Biot (1956a; 1956b); who modelled the binary continuum as a superposition of a fluid and a solid phase occupying simultaneously the same regions of space. Using the postulates of the linearised theory, Biot constructed his simplest model by assuming an isotropic, linear elastic soil skeleton saturated by a nondissipative compressible fluid (that is, a perfect fluid), which obey the following constitutive equations:

$$
\begin{aligned}
\mathbf{T}^{\mathrm{S}}= & G \cdot\left(\operatorname{grad} \mathbf{u}^{S}+\operatorname{grad}^{\mathrm{T}} \mathbf{u}^{\mathrm{S}}\right) \\
& +\left[(H-2 G) \cdot \mathbf{1} \cdot \operatorname{div} \mathbf{u}^{S}-C \cdot n \cdot \mathbf{1} \cdot \operatorname{div}\left(\mathbf{u}^{\mathrm{S}}-\mathbf{u}^{\mathrm{F}}\right)\right] \\
\mathbf{T}^{\mathrm{F}}= & p^{\mathrm{F}} \mathbf{1}=M \cdot n \cdot \mathbf{1} \cdot \operatorname{div}\left(\mathbf{u}^{\mathrm{S}}-\mathbf{u}^{\mathrm{F}}\right)-C \cdot \mathbf{1} \cdot \operatorname{div} \mathbf{u}^{\mathrm{S}}
\end{aligned}
$$

where $\mathbf{T}^{\mathrm{S}}$ and $\mathbf{T}^{\mathrm{F}}$ are the partial stress tensors of the soil skeleton and fluid phase respectively, $\mathbf{u}^{\mathrm{S}}$ and $\mathbf{u}^{\mathrm{F}}$ are the corresponding displacement vectors, $p^{\mathrm{F}}$ is the pressure of the pore fluid, $n$ is the porosity of the medium, $\mathbf{1}$ is the identity matrix, the notation $[\ldots]^{\mathrm{T}}$ denotes the transpose operator, $G$ is the elastic shear modulus of the soil skeleton, and Biot's elastic constants $H, C$ and $M$ are defined by the following relations (Stoll, 1989):

$$
\begin{aligned}
H & =\left(K^{\mathrm{S}}-K^{\mathrm{SK}}\right)^{2} /\left(D-K^{\mathrm{SK}}\right)+K^{\mathrm{SK}}+4 G / 3 \\
C & =K^{\mathrm{S}}\left(K^{\mathrm{S}}-K^{\mathrm{SK}}\right) /\left(D-K^{\mathrm{SK}}\right) \\
M & =\left(K^{\mathrm{S}}\right)^{2} /\left(D-K^{\mathrm{SK}}\right) \\
D & =K^{\mathrm{S}}\left[1+n \cdot\left(K^{\mathrm{S}} / K^{\mathrm{F}}-1\right)\right]
\end{aligned}
$$

where $K^{\mathrm{S}}$ is the bulk modulus of the soil grains, $K^{\mathrm{F}}$ is the bulk modulus of the pore fluid, and $K^{\mathrm{SK}}$ is the bulk modulus of the soil skeleton.

By invoking the balance law of linear momentum for each constituent of the mixture, and neglecting body forces and the non-linear contribution of the diffusion velocity (Bowen, 1982), constitutive equations (1) allow one to obtain the following equations of motion:

$$
\begin{aligned}
& \mathrm{G}\left(\nabla^{2} \mathbf{u}^{\mathrm{S}}\right.\left.+\operatorname{grad} \operatorname{div} \mathbf{u}^{\mathrm{S}}\right)+(H-2 G) \operatorname{grad} \operatorname{div} \mathbf{u}^{\mathrm{S}} \\
&-C \cdot n \cdot \operatorname{grad} \operatorname{div}\left(\mathbf{u}^{\mathrm{S}}-\mathbf{u}^{\mathrm{F}}\right)+\hat{\mathbf{p}}^{\mathrm{S}}=\rho^{\mathrm{S}}(1-n) \ddot{\mathbf{u}}^{\mathrm{S}} \\
& M \cdot n \cdot \operatorname{grad} \operatorname{div}\left(\mathbf{u}^{\mathrm{S}}-\mathbf{u}^{\mathrm{F}}\right)-C \cdot \operatorname{grad} \operatorname{div} \mathbf{u}^{\mathrm{S}}+\hat{\mathbf{p}}^{\mathrm{F}}=\rho^{\mathrm{F}} \cdot n \cdot \ddot{\mathbf{u}}^{\mathrm{F}}
\end{aligned}
$$

where $\nabla^{2}(\cdots)$ is the Laplacian operator, $\rho^{S}$ and $\rho^{\mathrm{F}}$ are respectively the mass densities of soil particles and pore fluid, and $\hat{\mathbf{p}}^{\alpha}(\alpha=\mathrm{S}, \mathrm{F})$ is the momentum supply vector of the $\alpha$ th component of the mixture, which accounts for the local interaction of the two constituents of the mixture. It is defined as the rate of increase, per unit mass of the mixture, of momentum of the $\alpha$ th constituent (for example the soil skeleton) due to interaction with the other (for example the pore fluid) (Bowen, 1982). The compatibility of equations (3) with the balance of linear momentum for the mixture as a whole requires that $\hat{\mathbf{p}}^{\mathrm{S}}=-\hat{\mathbf{p}}^{\mathrm{F}}$. Finally, in equations (3) the dot notation for the displacement vectors $\mathbf{u}^{\mathrm{S}}$ and $\mathbf{u}^{\mathrm{F}}$ is used to denote time differentiation.
Originally, Biot's equations of motion included a term, $\rho^{\mathrm{a}}$, called the apparent mass density, which was introduced to account for the inertial coupling caused by the relative motion between the solid and fluid phases (Biot, 1956a). The value of $\rho^{\mathrm{a}}$ is a function of the topology of a porous network through a parameter called tortuosity (Coussy, 1995; Gajo, 1996). However, more recent formulations of porous media theory have shown that the concept of apparent mass density is not only unnecessary (Bowen, 1982; Wilmanski, 1998), but actually violates the fundamental principle of material frame indifference (Wilmanski, 2001).

Since the porous medium is assumed to be isotropic, the rotational and dilatational motions are uncoupled, and they obey two independent wave equations (Biot, 1956a). By postulating that no relative motion occurs between the solid and the fluid phases (that is, $\mathbf{u}^{\mathrm{S}}=\mathbf{u}^{\mathrm{F}}=\mathbf{u}$ ), from equations (3) using Helmholtz's standard decomposition, it is possible to obtain

$$
\begin{aligned}
\nabla^{2}(H \cdot \operatorname{div} \mathbf{u}) & =\frac{\partial^{2}}{\partial t^{2}}(\rho \cdot \operatorname{div} \mathbf{u}) \\
\nabla^{2}(G \cdot \operatorname{curl} \mathbf{u}) & =\frac{\partial^{2}}{\partial t^{2}}(\rho \cdot \operatorname{curl} \mathbf{u})
\end{aligned}
$$

where $\rho$ is the bulk mass density of the porous medium, defined by

$$
\rho=(1-n) \rho^{\mathrm{S}}+n \rho^{\mathrm{F}}
$$

Equations (4) represent two wave equations governing respectively the propagation of the dilatational wave of the first kind, or P1-wave, and of the shear- or S-wave in a fluid-saturated porous medium, where it is assumed that relative motion between the fluid and solid phases is prevented. From equations (4) it is immediately possible to deduce the speed of propagation of these waves, here denoted by $V_{\mathrm{P}}$ and $V_{\mathrm{S}}$ respectively:

$$
\begin{aligned}
& V_{\mathrm{P}}=\sqrt{\frac{H}{\rho}} \\
& V_{\mathrm{S}}=\sqrt{\frac{G}{\rho}}
\end{aligned}
$$

where $H$ is Biot's coefficient defined by equations (2) and $G$ is the elastic shear modulus of the soil skeleton.

From an experimental point of view, the arrival times of the $\mathrm{P}$-wave and the $\mathrm{S}$-wave are relatively easy to detect, and in fact the determination of $V_{\mathrm{P}}$ and $V_{\mathrm{S}}$ constitutes in itself the main goal of the seismic prospecting methods used in geophysics and geotechnical engineering. However, as mentioned in the introduction, in the usual practice the measured values of $V_{\mathrm{P}}$ and $V_{\mathrm{S}}$ are used only to determine the corresponding stiffness parameters associated with a onecomponent linear elastic medium. In the next section it will be shown how, in a fluid-saturated medium, the measured values of $V_{\mathrm{P}}$ and $V_{\mathrm{S}}$ can also be used to determine porosity.

It is important to emphasise that equations (6) were obtained under the assumption that no relative motion occurs between the solid and the fluid phases: that is, that $\left(\mathbf{u}^{\mathrm{S}}-\mathbf{u}^{\mathrm{F}}\right)=0$. This condition is satisfied if the saturated porous medium is excited at low frequencies. In this case it behaves like a closed (undrained) system. The following relationship is helpful in identifying a characteristic frequency, $\omega_{\mathrm{c}}$, that can be considered as an estimate of the frequency upper bound for the validity of the assumption $\mathbf{u}^{\mathrm{S}}=\mathbf{u}^{\mathrm{F}}=\mathbf{u}$ (Miura et al., 2001): 


$$
\omega_{\mathrm{c}}=\frac{n g}{k}
$$

where $k$ is the coefficient of permeability of the medium, and $g$ is the acceleration due to gravity. Equation (7) shows that the main factor controlling the characteristic frequency is the coefficient of permeability. Table 1 shows calculations of $\omega_{\mathrm{c}}$ and $f_{\mathrm{c}}=\omega_{\mathrm{c}} / 2 \pi$ for typical values assumed in geomaterials by the parameters $n$ and $k$. The lowest characteristic frequency is that associated with gravelly soils, for which $f_{\mathrm{c}}$ is of the order of $70 \mathrm{~Hz}$.

A fundamental postulate laid at the base of Biot's theory of porous media is the assumption of continuity. Its validation imposes a restriction on the frequencies of the disturbances that can be propagated. For instance, in sandy formations dilatational waves of the first kind should propagate at frequencies smaller than about $3.5 \times 10^{5} \mathrm{~Hz}$ in order to avoid wavelengths of the same order of magnitude of the grain size (Miura et al., 2001). As shown in Table 1, this limiting value of frequency is about one order of magnitude higher than the corresponding characteristic frequency, $f_{\mathrm{c}}$. In silts and clays the situation is different, and the limiting frequency for the continuity assumption to hold approaches the same order of magnitude as that of $f_{\mathrm{c}}$.

\section{DETERMINING POROSITY FROM MEASURED $V_{\mathrm{P}}$ AND $V_{\mathrm{S}}$}

A procedure will now be presented for determining porosity in fluid-saturated media from the measured values of $V_{\mathrm{P}}$ and $V_{\mathrm{S}}$. The method is based on the application of Biot's theory under the assumption that no relative movement occurs in the porous medium between the solid and the fluid phases. This condition is considered to be fulfilled at low frequencies: that is, if the saturated porous medium is excited at frequencies less than $f_{\mathrm{c}}$.

Substitution of equations (2a) and (2d) in equation (6a) yields

$$
\rho V_{\mathrm{P}}^{2}-\left[K^{\mathrm{SK}}+\frac{4}{3} G\right]-\frac{\left(1-\frac{K^{\mathrm{SK}}}{K^{\mathrm{S}}}\right)^{2}}{\left[\frac{n}{K^{\mathrm{F}}}+\frac{(1-n)}{K^{\mathrm{S}}}-\frac{K^{\mathrm{SK}}}{\left(K^{\mathrm{S}}\right)^{2}}\right]}=0
$$

Now, since a perfect (that is, non-dissipative or inviscid) fluid cannot sustain shear stresses, the shear modulus $G^{\mathrm{SK}}$ of the (evacuated) soil skeleton is not different from the shear modulus $G$. Accordingly, it is possible to write the following identities:

$$
\begin{aligned}
\left(V_{\mathrm{S}}^{\mathrm{SK}}\right)^{2} & =\left[\frac{\rho}{\rho^{\mathrm{SK}}}\right] V_{\mathrm{S}}^{2} \\
\left(V_{\mathrm{P}}^{\mathrm{SK}}\right)^{2} & =\left[\frac{2\left(1-v^{\mathrm{SK}}\right)}{\left(1-2 v^{\mathrm{SK}}\right)}\right]\left[\frac{\rho}{\rho^{\mathrm{SK}}}\right] V_{\mathrm{S}}^{2} \\
K^{\mathrm{SK}} & =\left[\frac{2\left(1-v^{\mathrm{SK}}\right)}{\left(1-2 v^{\mathrm{SK}}\right)}-\frac{4}{3}\right] \rho \cdot V_{\mathrm{S}}^{2}
\end{aligned}
$$

where $V_{\mathrm{S}}^{\mathrm{SK}}$ and $V_{\mathrm{P}}^{\mathrm{SK}}$ are respectively the shear and dilatational wave velocity of the (evacuated) soil skeleton, and $K^{\mathrm{SK}}$ and $v^{\mathrm{SK}}$ are respectively the bulk modulus and Poisson ratio of the same medium phase. Finally, the term $\rho^{\mathrm{SK}}$ denotes the so-called $d r y$ mass density of the soil skeleton, which is given by the following relation:

$$
\rho^{\mathrm{SK}}=\rho^{\mathrm{S}}(1-n)
$$

Substitution of equations (6b) and (11) in equation (8) yields

$$
\begin{aligned}
& {\left[n\left(\rho^{\mathrm{F}}-\rho^{\mathrm{S}}\right)+\rho^{\mathrm{S}}\right]\left[V_{\mathrm{P}}^{2}-2\left(\frac{1-v^{\mathrm{SK}}}{1-2 v^{\mathrm{SK}}}\right) V_{\mathrm{S}}^{2}\right]} \\
& \left\{1-\frac{V_{\mathrm{S}}^{2}\left[n\left(\rho^{\mathrm{F}}-\rho^{\mathrm{S}}\right)+\rho^{\mathrm{S}}\right] \cdot\left[2\left(\frac{1-v^{\mathrm{SK}}}{1-2 v^{\mathrm{SK}}}\right)-\frac{4}{3}\right]}{K^{\mathrm{S}}}\right\}^{2} \\
& \left\{\frac{n}{K^{\mathrm{F}}}+\frac{(1-n)}{K^{\mathrm{S}}}-\frac{V_{\mathrm{S}}^{2}\left[n\left(\rho^{\mathrm{F}}-\rho^{\mathrm{S}}\right)+\rho^{\mathrm{S}}\right]\left[2\left(\frac{1-v^{\mathrm{SK}}}{1-2 v^{\mathrm{SK}}}\right)-\frac{4}{3}\right]}{\left(K^{\mathrm{S}}\right)^{2}}\right\}
\end{aligned}
$$

Formally, equation (13) is equivalent to the following nonlinear functional relationship:

$$
\mathscr{F}\left\{n, \rho^{\mathrm{F}}, \rho^{\mathrm{S}}, K^{\mathrm{F}}, K^{\mathrm{S}}, v^{\mathrm{SK}}, V_{\mathrm{P}}, V_{\mathrm{S}}\right\}=0
$$

It is useful to note the role played by each one of the eight variables upon which the function $\mathscr{F}$ is dependent:

(a) $V_{\mathrm{P}}, V_{\mathrm{S}}$ are supposed to be measured experimentally.

(b) $\rho^{\mathrm{F}}, \rho^{\mathrm{S}}, K^{\mathrm{F}}, K^{\mathrm{S}}$ are physical constants that assume standard numerical values (Carmichael, 1989).

(c) $v^{\mathrm{SK}}$ is a priori unknown, but its range of variability is limited to $0 \cdot 10-0 \cdot 40$ (Salem, 2000).

(d) $n$ is a priori unknown, and in this work it is considered to be the parameter of interest.

In light of these considerations, equation (14) can successfully be inverted for the porosity $n$ only on condition that the function $n=\mathscr{F}^{-1}\left\{v^{\mathrm{SK}}, \mathbf{d}\right\}$ is not too sensitive to the Poisson ratio of the (evacuated) soil skeleton. The argument $\mathbf{d}=\left[\rho^{\mathrm{F}}, \rho^{\mathrm{S}}, K^{\mathrm{F}}, K^{\mathrm{S}}, V_{\mathrm{P}}, V_{\mathrm{S}}\right]^{\mathrm{T}}$ in the above expression represents a vector of known data.

The sensitivity of the function $n=\mathscr{F}^{-1}\left\{v^{\mathrm{SK}}, \mathbf{d}\right\}$ to variations of the parameter $v^{\mathrm{SK}}$ (within the range $0 \cdot 10-0 \cdot 40$ ) was assessed through a series of numerical simulations. As expected, the function $n=\mathscr{F}^{-1}\left\{v^{\mathrm{SK}}, \mathbf{d}\right\}$ turned out to be

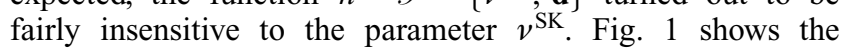

\begin{tabular}{|c|c|c|c|c|c|c|c|}
\hline Parameter & Loose sand & Dense sand & Silt & $\mathrm{NC}$ clay & OC clay & Gravel & Soft rock \\
\hline $\begin{array}{l}n \\
k: \mathrm{m} / \mathrm{s} \\
\omega_{\mathrm{c}}: \mathrm{rad} / \mathrm{s} \\
f_{\mathrm{c}}: \mathrm{Hz}\end{array}$ & $\begin{aligned} & 0.454 \\
& 1.0 \times 10^{-4} \\
& 4.45 \times 10^{4} \\
& 7.09 \times 10^{3}\end{aligned}$ & $\begin{array}{c}0.394 \\
1.0 \times 10^{-5} \\
3.86 \times 10^{5} \\
6.15 \times 10^{4}\end{array}$ & $\begin{array}{c}0.515 \\
1.0 \times 10^{-6} \\
5 \cdot 05 \times 10^{6} \\
8.04 \times 10^{5}\end{array}$ & $\begin{aligned} & 0.543 \\
& 1.0 \times 10^{-8} \\
& 5.33 \times 10^{8} \\
& 8.48 \times 10^{7}\end{aligned}$ & $\begin{aligned} & 0.429 \\
& 1 \cdot 0 \times 10^{-9} \\
& 4.21 \times 10^{9} \\
& 6.70 \times 10^{8} \\
&\end{aligned}$ & $\begin{array}{c}0.448 \\
1.0 \times 10^{-2} \\
4.39 \times 10^{2} \\
6.99 \times 10^{1} \\
\end{array}$ & $\begin{aligned} 0.315 \\
1.0 \times 10^{-7} \\
3.09 \times 10^{7} \\
4.92 \times 10^{6} \\
\end{aligned}$ \\
\hline
\end{tabular}
results for three different pairs of $\left\{V_{\mathrm{P}}, V_{\mathrm{S}}\right\}$. The numerical simulations were performed by solving the non-linear equation $\mathscr{F}\left\{n, v^{\mathrm{SK}}, \mathbf{d}\right\}=0$ for $n$ using the Newton-Raphson algorithm and assuming standard values for the physical constants $\left\{\rho^{\mathrm{F}}, \rho^{\mathrm{s}}, K^{\mathrm{F}}, K^{\mathrm{S}}\right\}$.

If soil particles are assumed to be incompressible, then in equation (13) $K^{\mathrm{S}} \rightarrow \infty$ and the relation $\mathscr{F}\left\{n, v^{\mathrm{SK}}, \mathbf{d}\right\}=0$ simplifies as follows:

Table 1. Characteristic frequencies for typical values of porosity and permeability in geomaterials (modified after Miura et al., 2001) 


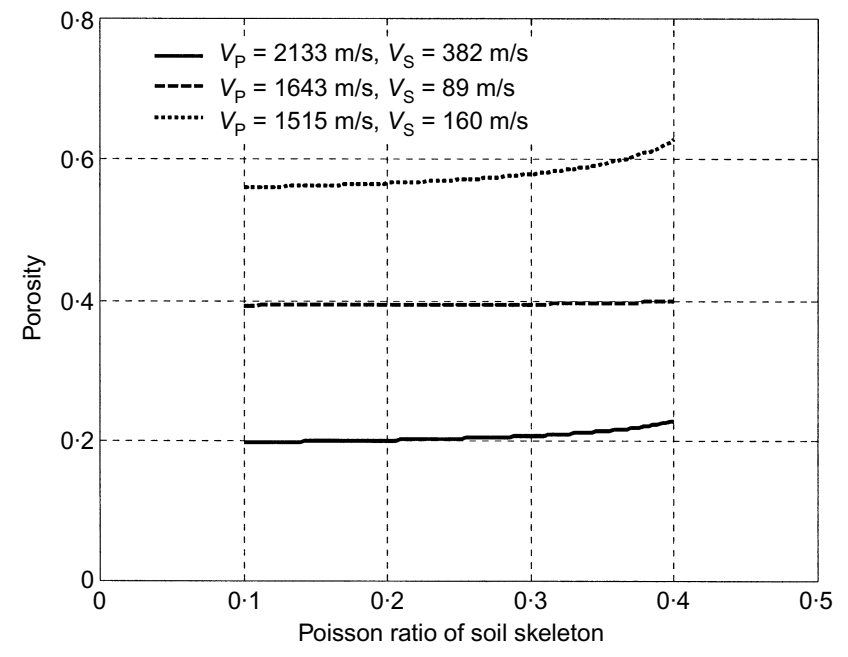

Fig. 1. Dependence of porosity on Poisson ratio of (evacuated) soil skeleton

$$
\left[n\left(\rho^{\mathrm{F}}-\rho^{\mathrm{S}}\right)+\rho^{\mathrm{S}}\right]\left[V_{\mathrm{P}}^{2}-2\left(\frac{1-v^{\mathrm{SK}}}{1-2 v^{\mathrm{SK}}}\right) V_{\mathrm{S}}^{2}\right]-\frac{K^{\mathrm{F}}}{n}=0
$$

which is a quadratic equation in $n$ that is trivial to solve.
Selecting the root that satisfies the admissibility condition $0 \leqslant n \leqslant 1$, equation (15) yields the following explicit relationship for $n$ :

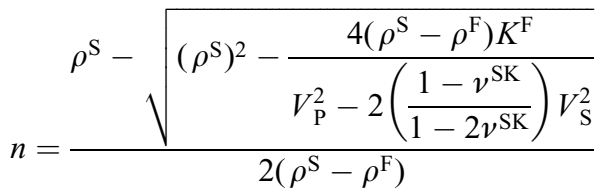

Figure 2 shows the difference between the results obtained from the solution of equation (13) (exact) and those obtained by applying equation (16) (approximate). The numerical simulation was conducted using the $V_{\mathrm{P}}$ and $V_{\mathrm{S}}$ profiles shown in Fig. 3, which were randomly generated with uniform distribution and with the constraint that the returned values of $V_{\mathrm{P}}$ and $V_{\mathrm{S}}$ would correspond to values of porosity in the range $0 \cdot 2-0 \cdot 6$. The parameters $v^{\mathrm{SK}}, \rho^{\mathrm{F}}, \rho^{\mathrm{S}}$, $K^{\mathrm{F}}, K^{\mathrm{S}}$ were assumed equal to $0 \cdot 25,1 \mathrm{t} / \mathrm{m}^{3}, 2.7 \mathrm{t} / \mathrm{m}^{3}$, $2.25 \times 10^{6} \mathrm{kPa}$ and $6.81 \times 10^{7} \mathrm{kPa}$ respectively.

Figure 2 shows that the assumption of neglecting the deformability of soil particles may lead to a slight to moderate overestimation of porosity. The minimum, maximum and average relative errors resulting from the above numerical simulation are respectively equal to $6 \cdot 7 \%, 16.5 \%$ and $9.8 \%$. Such errors can be considered acceptable from a practical point of view, and hence equation (16) constitutes a simple and rapid method for estimating porosity from measured values of $V_{\mathrm{P}}$ and $V_{\mathrm{S}}$.

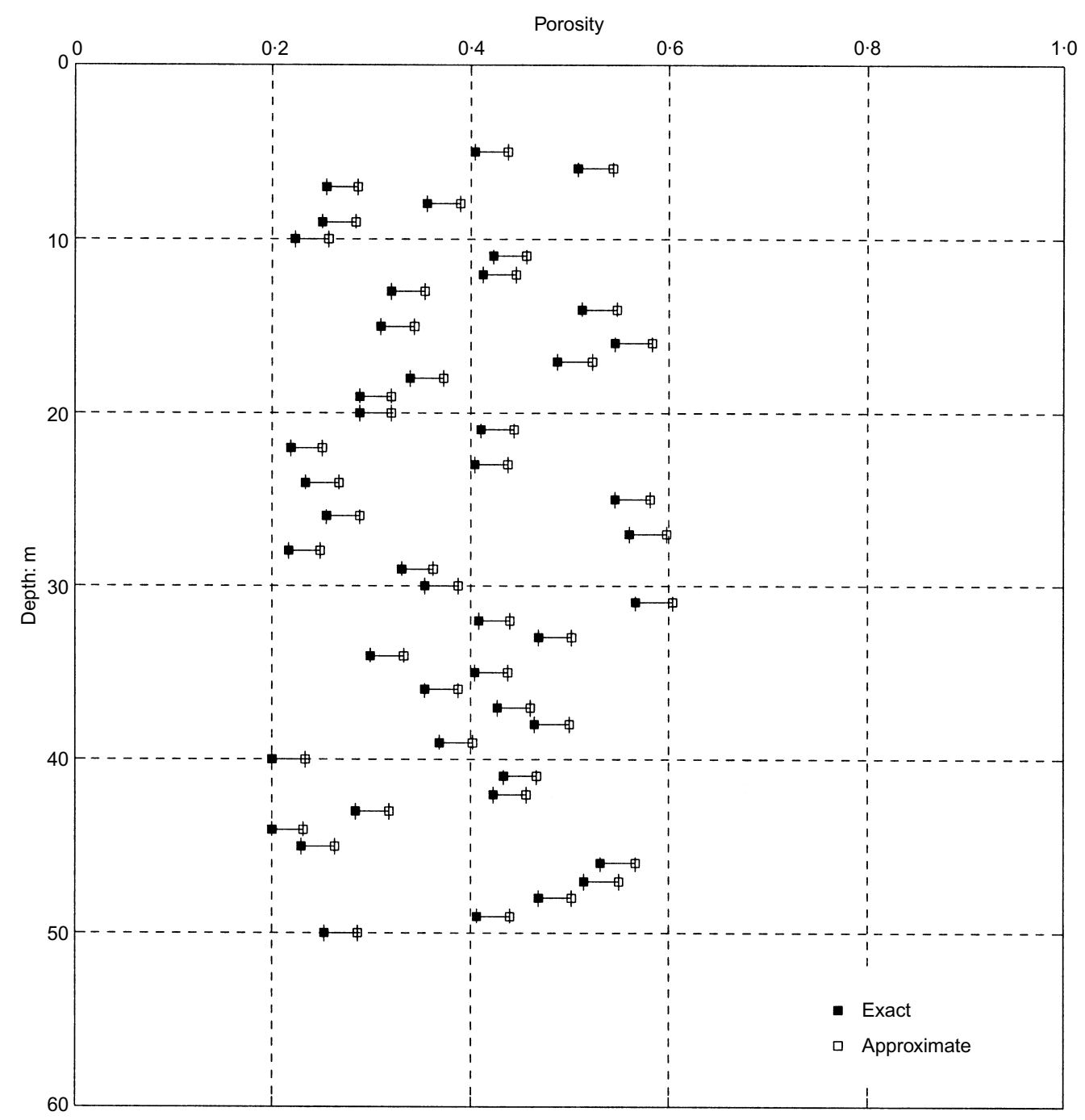

Fig. 2. Comparison between exact and approximate solutions in determining $n$ 


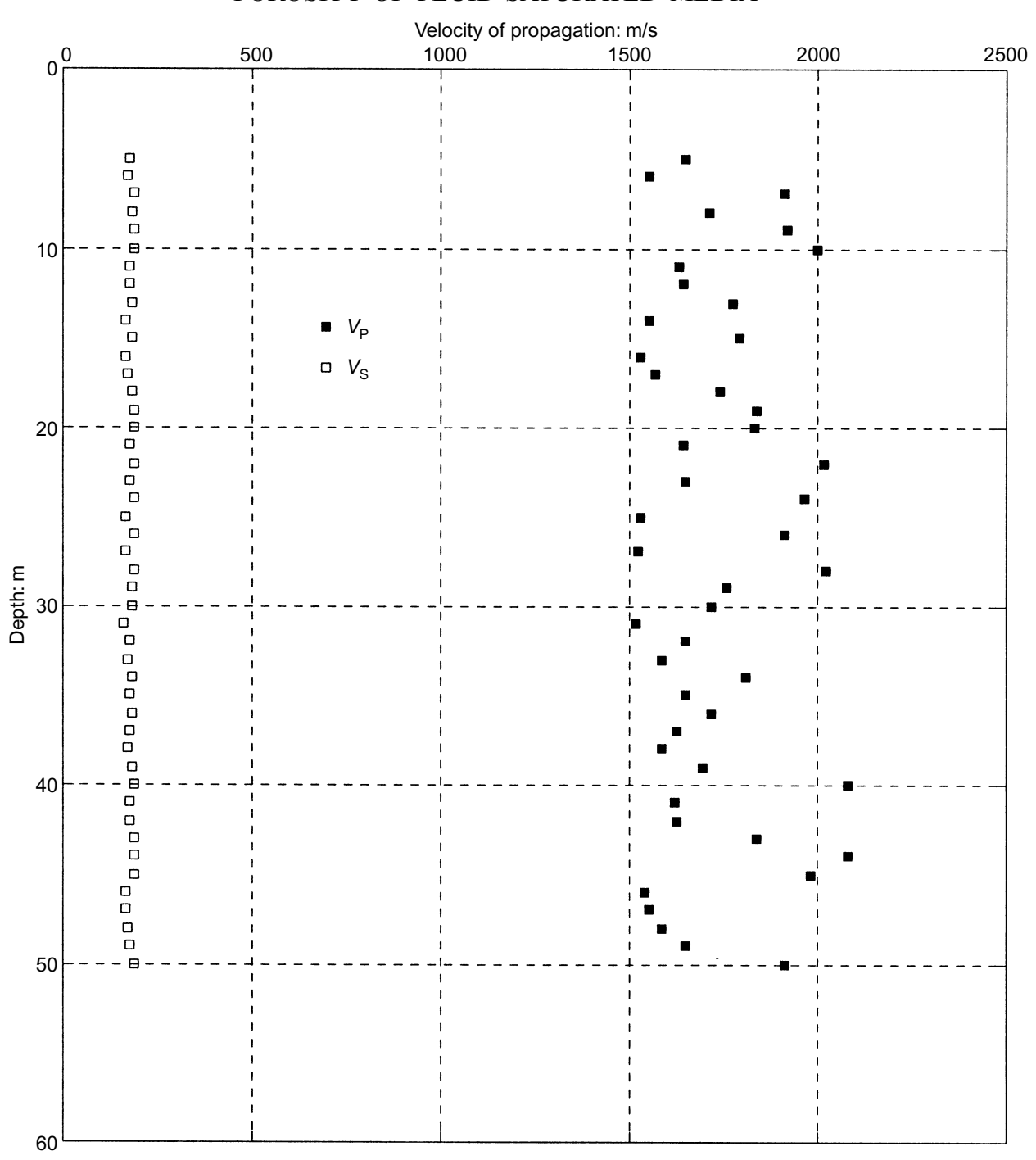

Fig. 3. Randomly generated $V_{\mathrm{P}}$ and $V_{\mathrm{S}}$ profiles used in the numerical simulations

If soil particles are assumed to be incompressible, the expression for the computation of $V_{\mathrm{P}}$ simplifies as follows:

$$
V_{\mathrm{P}}=\sqrt{\frac{\left(K^{\mathrm{SK}}+\frac{4}{3} G\right)+\frac{K^{\mathrm{F}}}{n}}{(1-n) \rho^{\mathrm{S}}+n \rho^{\mathrm{F}}}}
$$

\section{VALIDATION OF THE PROCEDURE USING LABORATORY DATA}

The velocity of propagation of seismic waves in geomaterials can be measured in laboratory tests using bender elements and piezoelectric crystals. These devices are placed inside a soil specimen, and they can act as source or receiver of shear and compressional waves (Dyvik \& Madshus, 1985; Lings \& Greening, 2001). Data from the literature (Bates, 1989) are herein used to assess the validity of the proposed method for the prediction of porosity. In the work of Bates (1989) the seismic wave velocities $V_{\mathrm{P}}$ and $V_{\mathrm{S}}$ were measured at different levels of confining pressure during an isotropic compression test performed on a sample of fine sand. The saturated bulk density of the sample was $\rho=1.987 \mathrm{~g} / \mathrm{cm}^{3}$. Assuming for the soil grains a mass density of $2.65 \mathrm{~g} / \mathrm{cm}^{3}$, the porosity was estimated to be about $0 \cdot 40$.

The seismic wave velocities reported in Bates (1989) were used to estimate the porosity under the assumption of incompressible grains (see Fig. 4). The bulk modulus of the fluid, $K^{\mathrm{F}}$, was computed from the relation $K^{\mathrm{F}}=\rho^{\mathrm{F}}\left(V_{\mathrm{P}}^{\mathrm{F}}\right)^{2}$, where $V_{\mathrm{P}}^{\mathrm{F}}=V_{\mathrm{P}}^{\mathrm{W}}$ is the velocity of propagation of sound in water, which in general is a function of temperature and pressure. The dependence of $V_{\mathrm{P}}^{\mathrm{W}}$ on pressure is of the order of $0.1 \%$ for the range of pressures applied during the test, and hence it can be neglected (Belogol'skii et al., 1999). The dependence of $V_{\mathrm{P}}^{\mathrm{W}}$ on temperature was estimated using the relation by Marczak (1997), the range of validity of which at atmospheric pressure is between 0 and $100^{\circ} \mathrm{C}$. It was assumed that the laboratory test was performed at a standard temperature of $20^{\circ} \mathrm{C}$.

Figure 5 shows a comparison between the porosity evaluated in the laboratory from the saturated bulk density and that obtained with the proposed method using measured $V_{\mathrm{P}}$ and $V_{\mathrm{S}}$. Overall, the agreement between the two estimates is good. For the computed values of porosity, the error bars show the confidence level of the prediction for a range of variability of the unknown parameter $v^{\mathrm{SK}}$ (the Poisson ratio of the evacuated soil skeleton) between $0 \cdot 10$ and $0 \cdot 30$. It is 


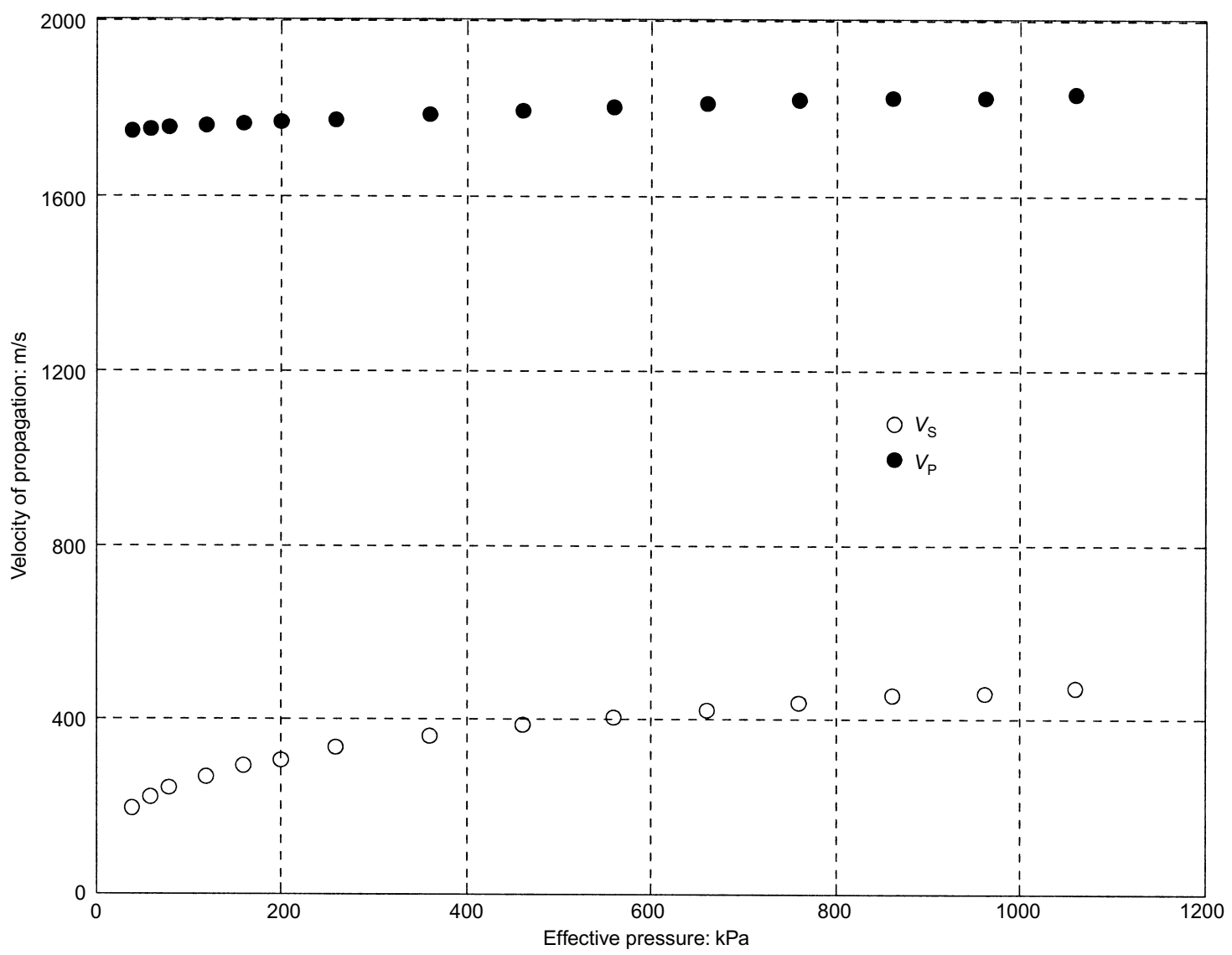

Fig. 4. Seismic wave velocities measured in laboratory on a specimen of saturated sand (data from Bates, 1989)

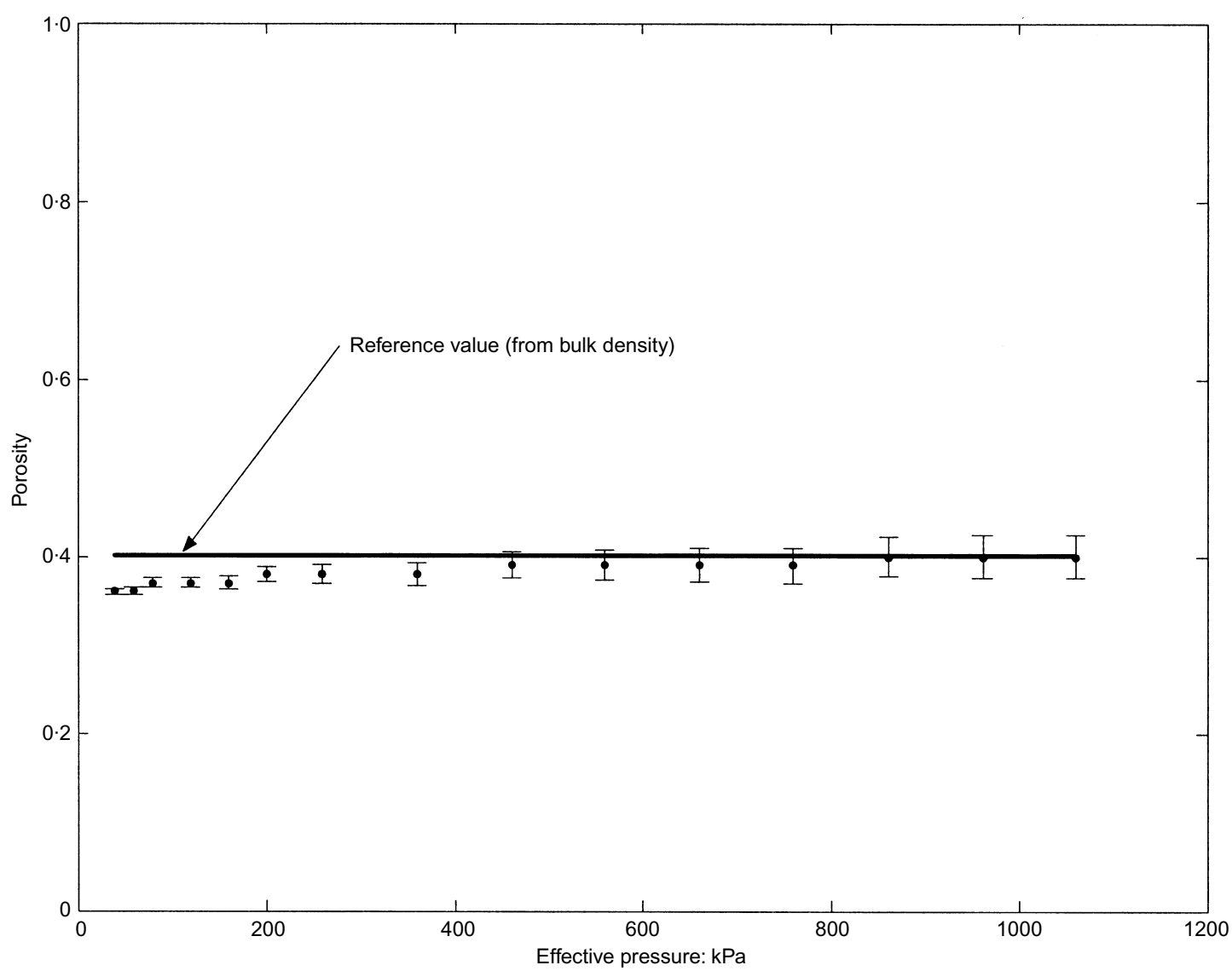

Fig. 5. Comparison of porosity estimated with the proposed method and the value measured in the laboratory using data from Bates (1989) 
interesting to note that the uncertainty associated with the unknown $v^{\mathrm{SK}}$ increases with increase of the confining pressure.

\section{SITE DESCRIPTIONS AND SEISMIC CROSS-HOLE MEASUREMENTS}

The procedure illustrated in the previous section for determining porosity from the measured values of $V_{\mathrm{P}}$ and $V_{\mathrm{S}}$ has been applied at two sites in Italy where cross-hole seismic data were available. The first site is located at Messina Strait in southern Italy, where a comprehensive geotechnical testing programme was conducted at the end of the 1980s in connection with the preliminary design of a single-span suspended bridge linking Sicily to Calabria (Stretto di Messina, 1989).

Table 2 shows the in-situ geotechnical investigation programme conducted at Messina Strait, which also included a series of seismic cross-hole tests conducted at depths down to $100 \mathrm{~m}$ below ground level. The subsoil conditions at both sides of the Messina Strait consist of sandy-gravelly deposits of Holocene and Pleistocene age underlain by soft rocks of Pliocene and Miocene age (Crova et al., 1992). The bedrock is located at depths exceeding $150 \mathrm{~m}$ below ground level.

Schematic representations of the soil profiles on the two sides of the Messina Strait are shown in Figs 6 and 7 together with the shear and compression wave velocity profiles measured from the seismic cross-hole tests performed at the foundation sites of the one-span suspended bridge. The reported values of $V_{\mathrm{P}}$ and $V_{\mathrm{S}}$ represent the mean of three independent measurements performed at each depth along three source-to-receiver travel paths between the boreholes (see Fig. 8).

Seismic cross-hole measurements were made using two source-to-receiver configurations. In the first configuration the source was placed in the central borehole (FD1 in Fig. 8 ) and the receivers were placed at the same depth in the other two boreholes (BH1 and SPT1 in Fig. 8). In the second configuration the source was placed in one of the two end boreholes (for example BH1 in Fig. 8) and the receivers were inserted, at the same depth, in the remaining two boreholes (that is, FD1 and SPT1 in Fig. 8). The spacing between the boreholes was set constant and equal to $5 \mathrm{~m}$. In each borehole a deviation survey was conducted to accurately determine the horizontal distance between borings along the depth. All measurements were made at a constant depth interval of $1 \mathrm{~m}$.

The soil profile at the Calabria foundation site consists of $25 \mathrm{~m}$ of loose to medium, non-cemented silty sand underlain up to a depth of $100 \mathrm{~m}$ by a layer of dense, fine to medium sand with gravel and occasionally cemented boulders. At the Sicily foundation site the subsoil conditions up to a depth of $100 \mathrm{~m}$ consist of sand and gravel of various grain sizes with the occasional presence of boulders. The top $72 \mathrm{~m}$ of this soil stratum belongs to the Holocene formation, whereas the deeper strata belong to the older, Pleistocene formation. The water table was detected at $0.75 \mathrm{~m}$ and $2.2 \mathrm{~m}$ below ground level of the Calabria and Sicily foundation sites respectively. Concerning laboratory soil classification tests, Figs 9 and 10 show the grain size distribution curves obtained from the samples retrieved at the two cross-hole testing sites.

The second site considered in this study is that associated with the Leaning Tower of Pisa. The subsoil conditions at this site have been thoroughly investigated during recent decades in view of the stabilisation works successfully completed recently. For the depths of interest considered in this study, it is possible to identify three main geological formations with ages spanning from the Holocene to the Pleistocene (Costanzo et al., 1995).

The first formation (A) is composed mainly of slightly clayey and sandy silt with lenses, and layers of sand and clay. At the bottom of this formation is a layer of medium uniform sands of variable thickness.

The second formation (B) is primarily clayey. It can be subdivided into four main layers:

(a) Upper Clay, also termed Pancone Clay

(b) Intermediate Clay

(c) Intermediate Sand

(d) Lower Clay.

The third formation (C) is a slightly silty sand that extends down to a depth of about $70 \mathrm{~m}$. At a depth of about $50 \mathrm{~m}$ there is a quite stiff, slightly cemented thin layer.

The index properties of the three formations are reported in Table 3. Fig. 11 shows a typical result of a CPT test and the estimated OCR profile in the first top $40 \mathrm{~m}$. The observed preconsolidation can be attributed to groundwater oscillations, secondary compression and, to a certain degree, diagenetic processes (Costanzo et al., 1995).

Figure 12 shows the measured values of $V_{\mathrm{P}}$ and $V_{\mathrm{S}}$ together with a simplified soil profile inferred from the

Table 2. Geotechnical investigation programme conducted at Messina Strait for preliminary design of the single-span suspension bridge (modified after Crova et al., 1992)

\begin{tabular}{l|c|c|c|c}
\hline \multirow{2}{*}{ Test } & \multicolumn{2}{|c|}{ Calabria } & \multicolumn{2}{c}{ Sicily } \\
\cline { 2 - 5 } & Foundation & Anchor block & Foundation & Anchor block \\
\hline BH* & 4 & 5 & 2 & 3 \\
SPT $\dagger$ & 7 & 6 & 7 & 6 \\
LPT & 5 & 5 & 5 & 5 \\
PLT & None & 3 & None & 3 \\
CPT & 1 & None & 3 & None \\
CHT§ & 2 & 1 & 2 & 1 \\
SASW & 1 & None & 1 & 1 \\
PMT & 1 & & 1 & None
\end{tabular}

BH, boring with sampling; SPT, standard penetration test; LPT, large penetration test; PLT, plate loading test; CPT, static cone penetration test; CHT, cross-hole test; SASW, spectral analysis of surface waves; PMT, pumping test.

$*$ Depth $=100 \mathrm{~m}$ in addition to a number of geological borings.

$\dagger$ Depth $=50 \mathrm{~m}$ with rod energy measurements.

tDepth $=18 \mathrm{~m}$ in $2.5 \mathrm{~m}$ diameter cased well, in-situ measurement of density

$\S$ Depth $=100 \mathrm{~m}$. 

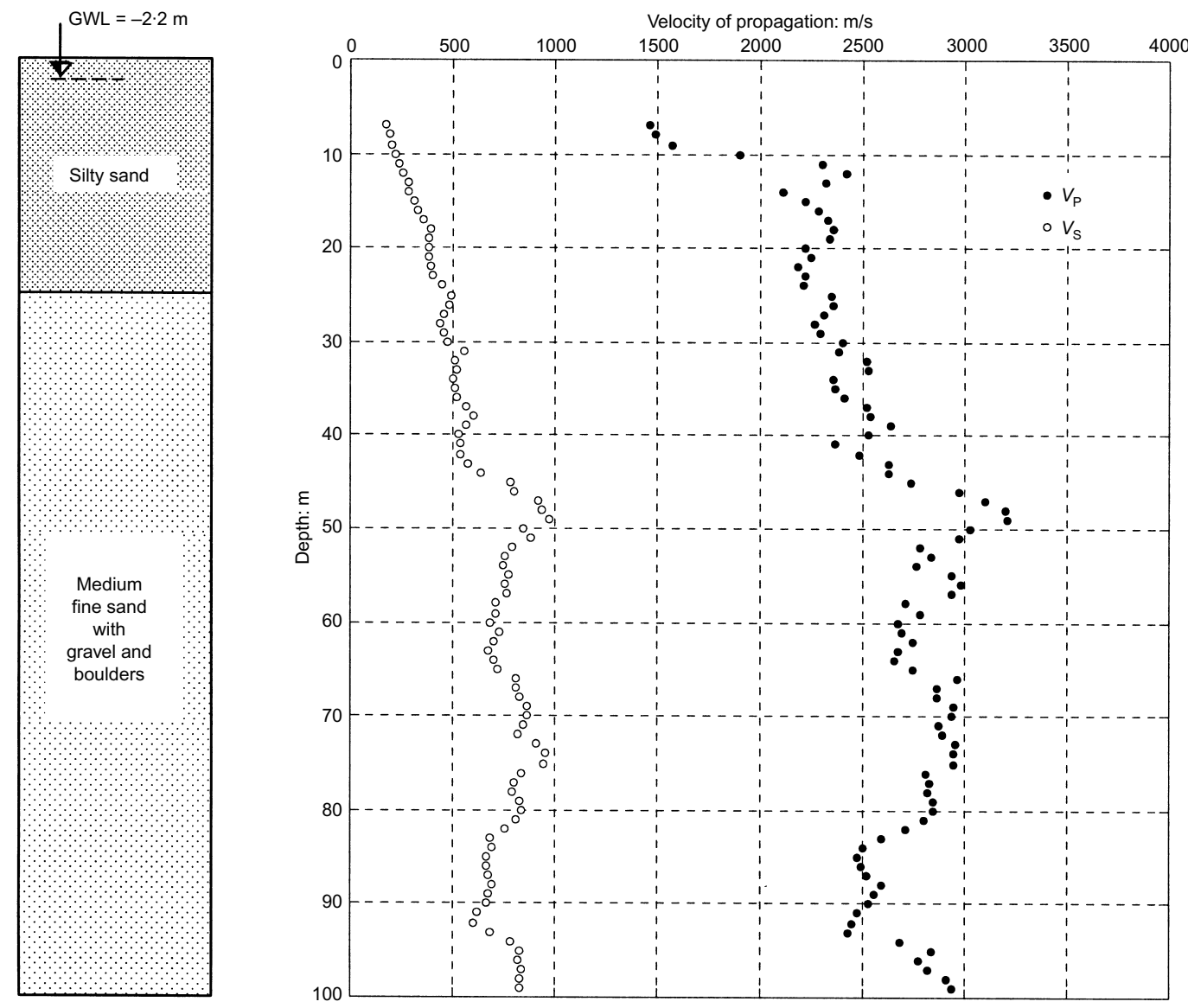

Fig. 6. Soil profile and $V_{P}$ and $V_{S}$ charts at Messina Strait, Calabria foundation site
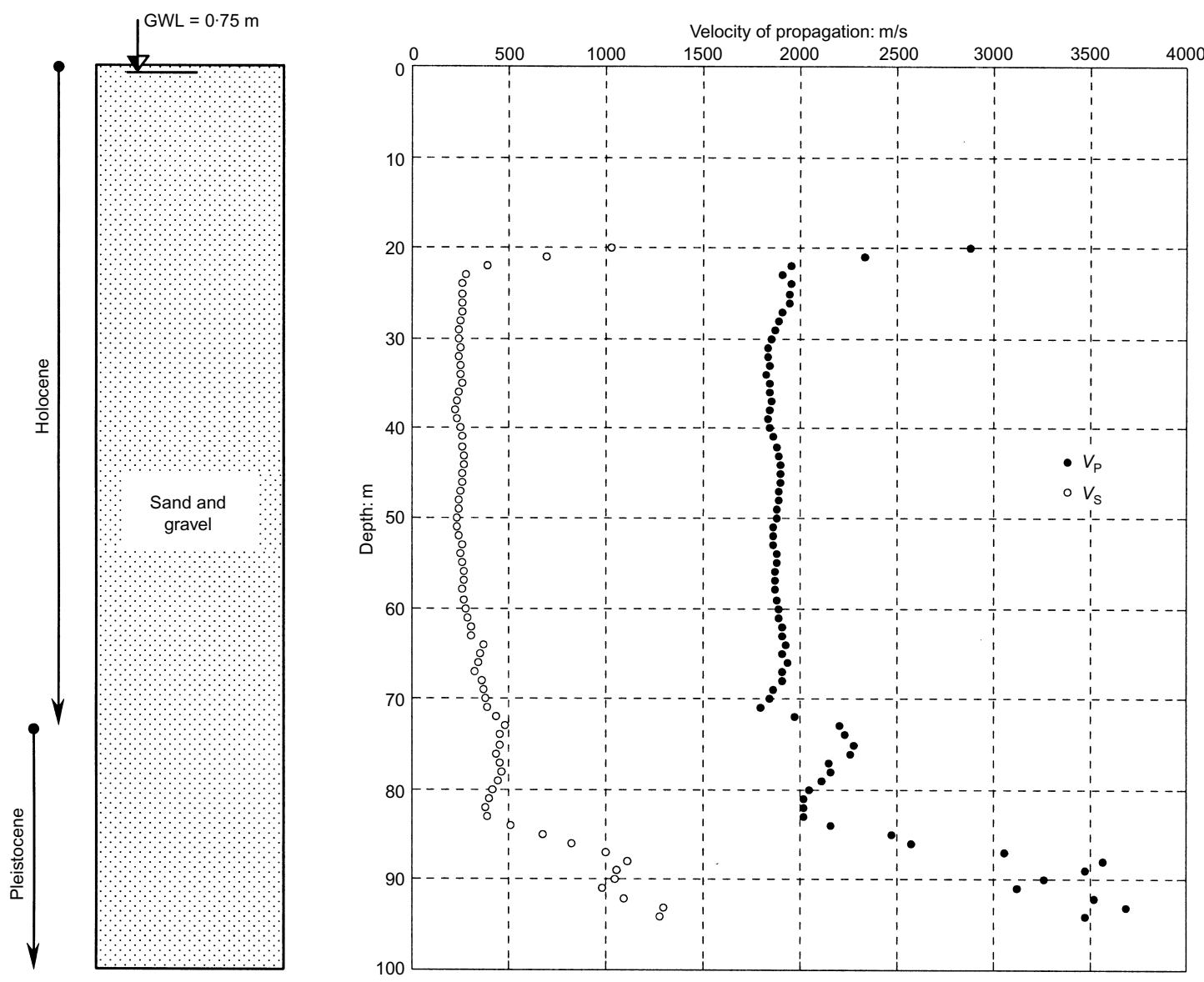

Fig. 7. Soil profile and $V_{\mathrm{P}}$ and $V_{\mathrm{S}}$ charts at Messina Strait, Sicily foundation site 


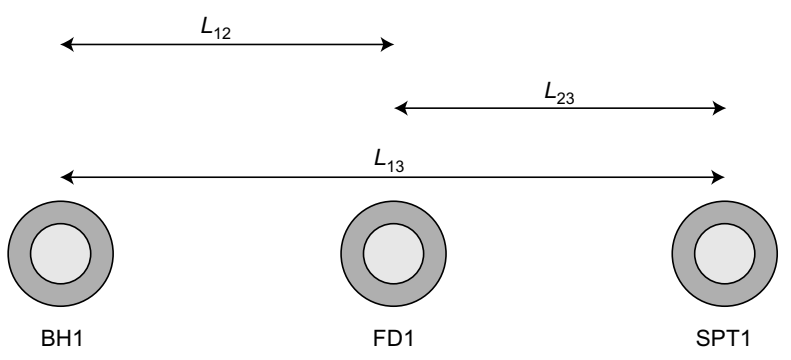

Fig. 8. Borehole layout used for cross-hole seismic testing at Messina Strait borehole logs. The cross-hole measurements have been performed using three boreholes, with the source located in one of the external boreholes. The values of $V_{\mathrm{P}}$ and $V_{\mathrm{S}}$ have been computed from the difference in travel time between the two receivers. The spacing between the boreholes was set constant and equal to $4 \mathrm{~m}$. Sonic measurements have been performed in each borehole to verify the contact conditions between the cementation of the internal tube and the surrounding soil. In each borehole a deviation survey was conducted to accurately determine the horizontal distance between borings along the depth. All measurements were made at a constant depth interval of $1 \mathrm{~m}$.

\begin{tabular}{|c|c|c|c|c|c|c|c|c|c|c|}
\hline \multirow{2}{*}{ Cobbles } & \multicolumn{3}{|c|}{ Gravel } & \multicolumn{3}{|c|}{ Sand } & \multicolumn{3}{|c|}{ Silt } & \multirow{2}{*}{ Clay } \\
\hline & G & $M$ & $\mathrm{~F}$ & G & $M$ & $\mathrm{~F}$ & $G$ & $M$ & $\mathrm{~F}$ & \\
\hline 6 & \multicolumn{2}{|c|}{20} & \multicolumn{2}{|c|}{$2 \cdot 0$} & \multicolumn{2}{|c|}{0.2} & \multicolumn{2}{|c|}{0.02} & & \\
\hline
\end{tabular}

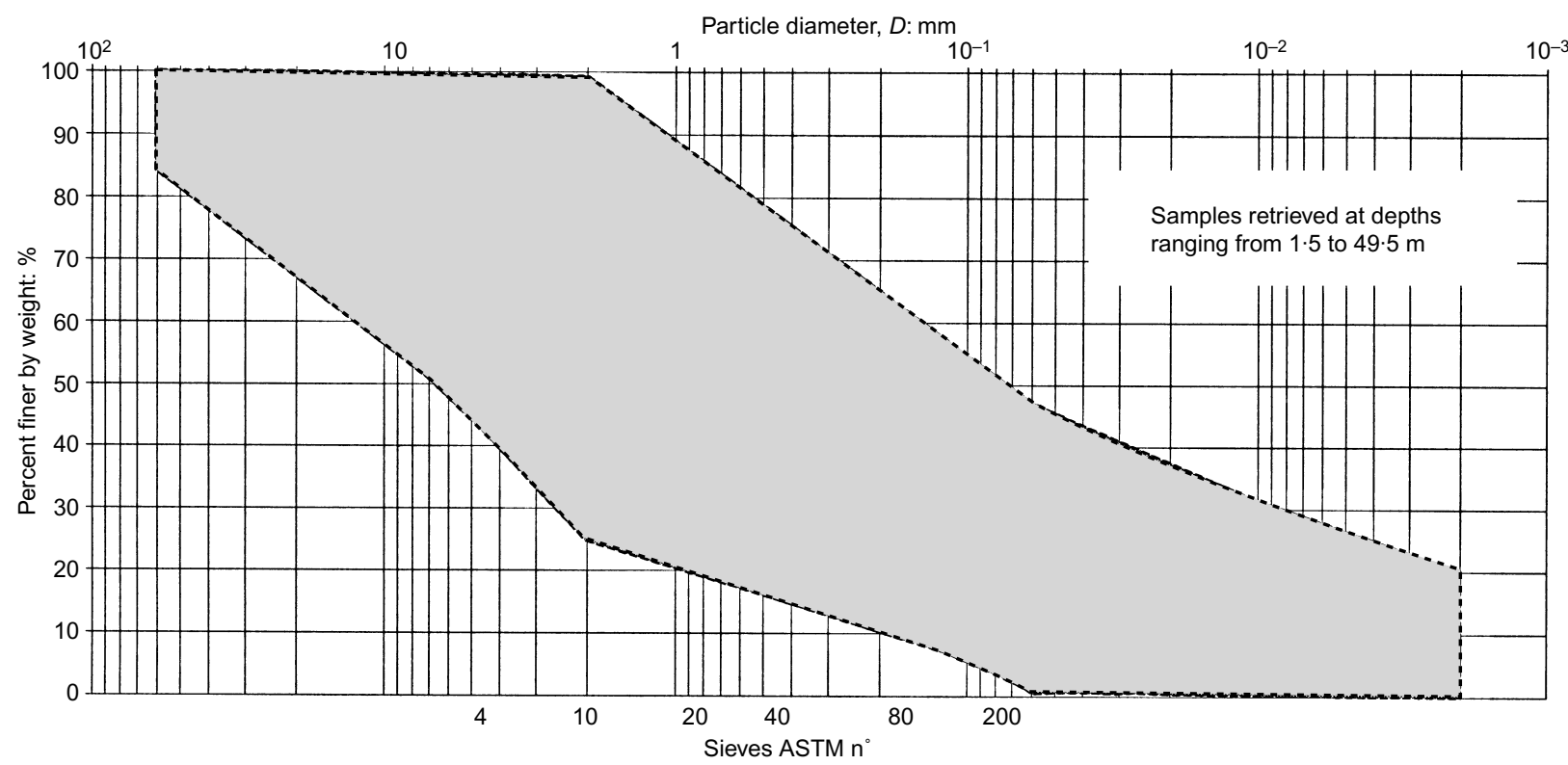

Fig. 9. Grain size distribution at Messina Strait, Calabria foundation site

\begin{tabular}{|c|c|c|c|c|c|c|c|c|c|c|}
\hline \multirow{2}{*}{ Cobbles } & \multicolumn{3}{|c|}{ Gravel } & \multicolumn{3}{|c|}{ Sand } & \multicolumn{3}{|c|}{ Silt } & \multirow{2}{*}{ Clay } \\
\hline & G & M & $\mathrm{F}$ & G & M & $\mathrm{F}$ & G & M & $\mathrm{F}$ & \\
\hline
\end{tabular}

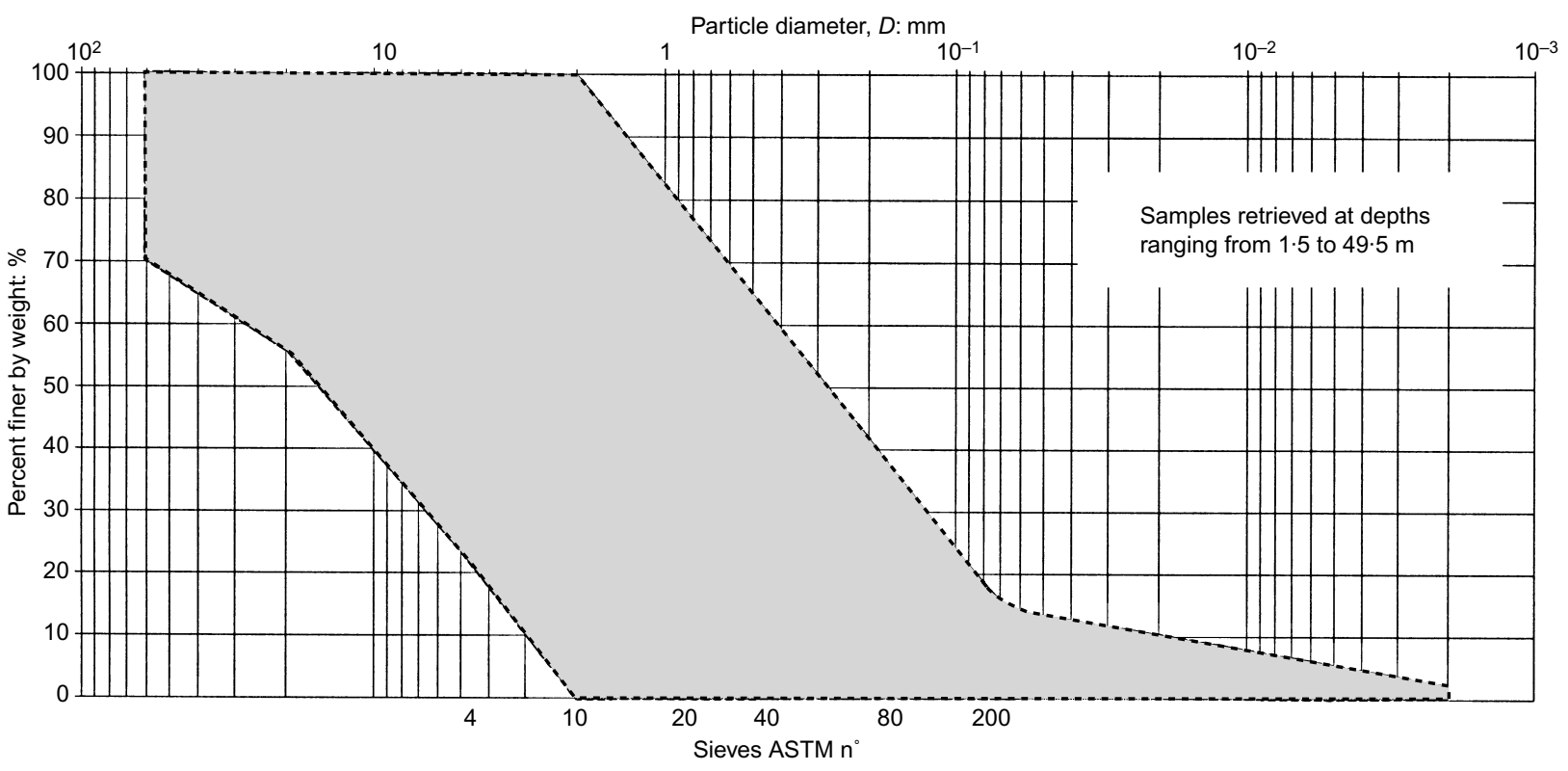

Fig. 10. Grain size distribution at Messina Strait, Sicily foundation site 
Table 3. Index properties of soils at Pisa site (after Costanzo et al., 1995)

\begin{tabular}{|c|c|c|c|c|c|c|}
\hline \multicolumn{2}{|c|}{ Formation } & $\gamma: \mathrm{kN} / \mathrm{m}^{3}$ & LL: \% & PI: \% & Fines: \% & $G_{\mathrm{S}}: \mathrm{kN} / \mathrm{m}^{3}$ \\
\hline \multicolumn{2}{|c|}{ A } & $18 \cdot 1-19 \cdot 0$ & $28-42^{*}$ & $8-19^{*}$ & $22-100$ & $26 \cdot 4-26 \cdot 9$ \\
\hline B & $\begin{array}{l}\mathrm{B}_{1} \\
\mathrm{~B}_{2} \\
\mathrm{~B}_{3} \\
\mathrm{~B}_{4}\end{array}$ & $\begin{array}{l}16 \cdot 4-17 \cdot 8 \\
19 \cdot 4-20 \cdot 4 \\
18 \cdot 5-19 \cdot 4 \\
17 \cdot 6-19 \cdot 3\end{array}$ & $\begin{array}{c}53-61 \\
34-61 \\
\mathrm{NP} \\
35-78\end{array}$ & $\begin{array}{c}27-57 \\
13-39 \\
\mathrm{NP} \\
17-48\end{array}$ & $\begin{array}{l}>80 \\
>80 \\
3-50 \\
>80\end{array}$ & $\begin{array}{l}27 \cdot 0-27 \cdot 3 \\
26 \cdot 8-27 \cdot 3 \\
26 \cdot 2-26 \cdot 4 \\
26 \cdot 3-26 \cdot 8\end{array}$ \\
\hline \multicolumn{2}{|l|}{$\mathrm{C}$} & $20 \cdot 2-21 \cdot 4$ & NP & NP & $0-20$ & $26 \cdot 0-26 \cdot 2$ \\
\hline
\end{tabular}

* Silt and clay layers.

$\mathrm{NP}=$ non-plastic.

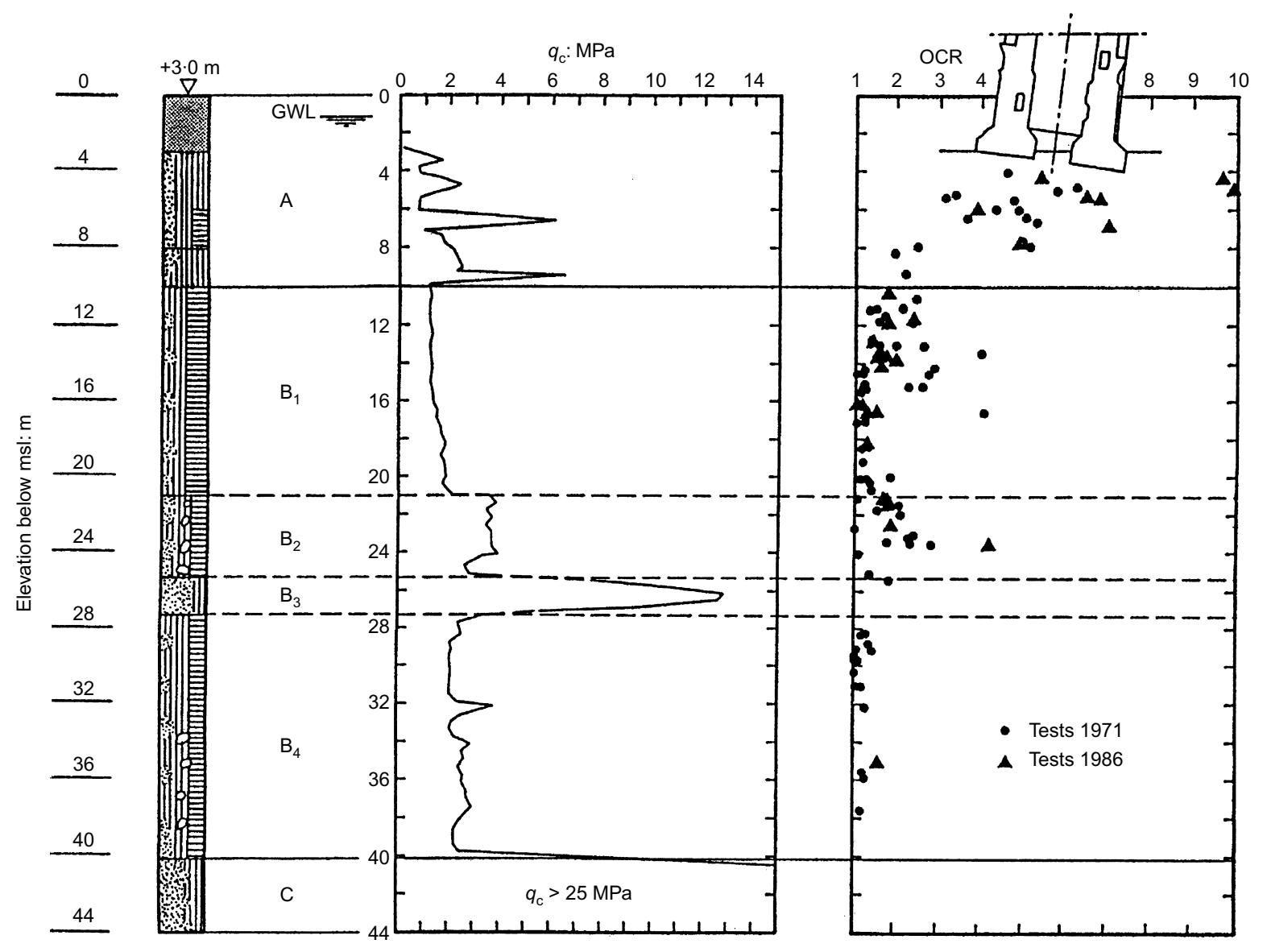

Fig. 11. CPT results and OCR profile at Pisa site (after Costanzo et al., 1995)

\section{RESULTS AND DISCUSSION}

Using the values of $V_{\mathrm{P}}$ and $V_{\mathrm{S}}$ measured experimentally at the Messina Strait and Pisa sites from cross-hole seismic tests, equation (13) has been solved for the porosity, $n$. For the physical constants $\rho^{\mathrm{F}}, \rho^{\mathrm{S}}, K^{\mathrm{F}}$ and $K^{\mathrm{S}}$ the values reported in Table 4 were assumed. Soil grains were assumed to be incompressible at both sites owing to the difficulties of making a correct estimate of $K^{\mathrm{S}}$ in soil deposits characterised by a varying mineralogical composition and a high degree of micro-heterogeneity.

Figures 13 and 14 show the computed porosity profile at the Messina Strait sites. The error bars attached to the computed values of $n$ show the confidence level of the prediction for an assumed range of variability of the un- known parameter $v^{\mathrm{SK}}$ (the Poisson ratio of the evacuated soil skeleton) between $0 \cdot 10$ and $0 \cdot 35$. The figures show that the predicted porosity is, in general, not very sensitive to variations of the parameter $v^{\mathrm{SK}}$, confirming a result that was already anticipated in the numerical simulations.

Figure 13 shows that for depths ranging from $7 \mathrm{~m}$ to $40 \mathrm{~m}$ only a pair of computed values of $n$, both located at shallow depths, are affected by a relatively large uncertainty. At greater depths the uncertainty is small to moderate. Fig. 14, by contrast, shows that for depths between $23 \mathrm{~m}$ and about $63 \mathrm{~m}$ the predicted values of porosity are almost independent of the assumed value of $v^{\mathrm{SK}}$. The average values of $n$ predicted at the Calabria foundation site (see Fig. 13), vary for depths between $10 \mathrm{~m}$ and $100 \mathrm{~m}$ from 0.11 to about 

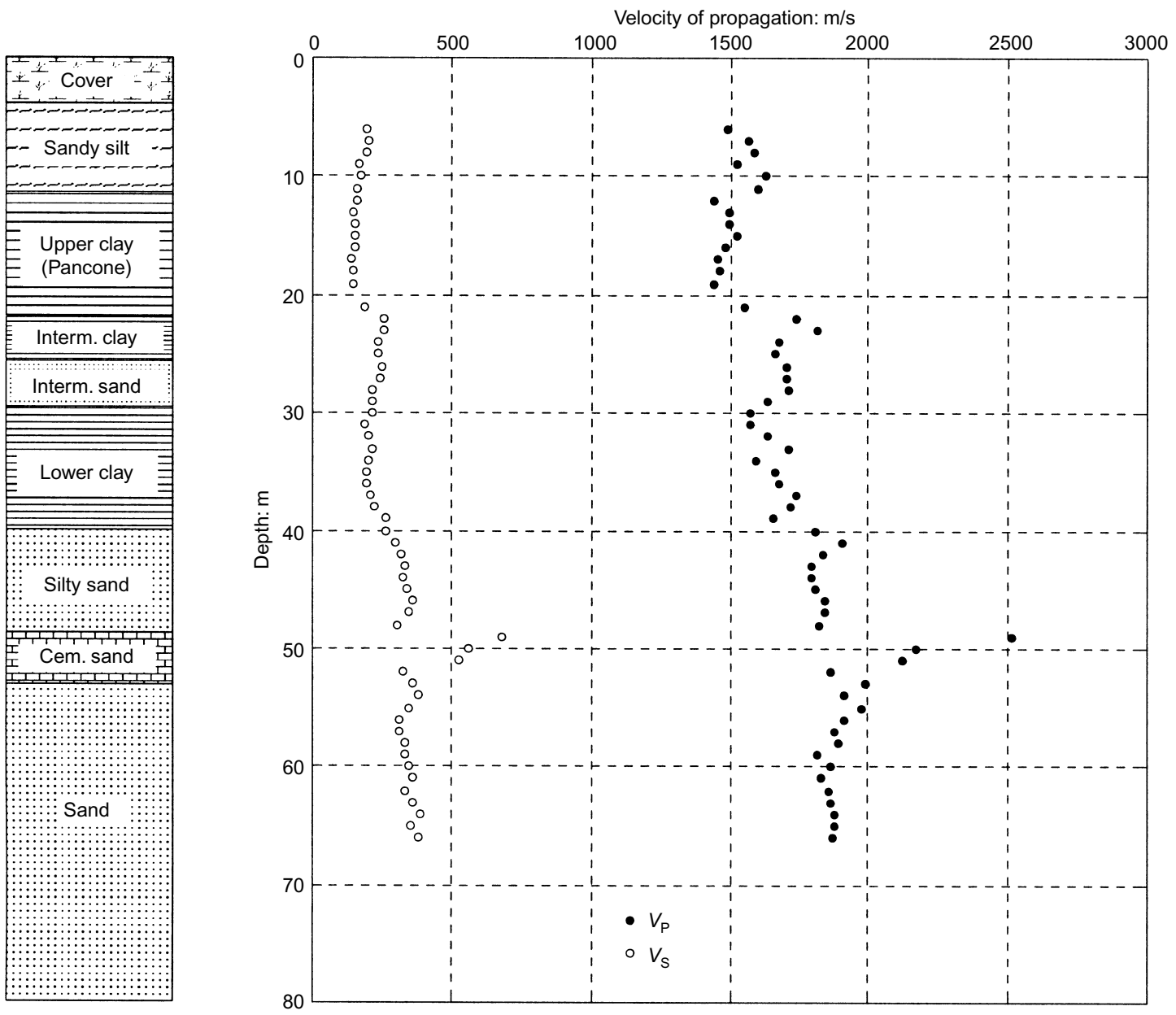

Fig. 12. Soil profile and $V_{\mathrm{P}}$ and $V_{\mathrm{S}}$ charts at Pisa site

Table 4. Values assumed for the physical constants for determining porosity at the two testing sites

\begin{tabular}{l|c|c|c|c}
\hline \multirow{2}{*}{ Testing site } & \multicolumn{4}{|c}{ Physical property } \\
\cline { 2 - 5 } & $\rho^{\mathrm{F}}: \mathrm{Mg} / \mathrm{m}^{3}$ & $\rho^{\mathrm{S}}: \mathrm{Mg} / \mathrm{m}^{3}$ & $K^{\mathrm{S}}: \mathrm{kPa}$ & $K^{\mathrm{F}}: \mathrm{kPa}$ \\
\hline Messina Strait & 1 & $2 \cdot 65$ & $\infty$ & $*$ \\
Calabria foundation site & 1 & $2 \cdot 65$ & $\infty$ & $*$ \\
Sicily foundation site & 1 & $2 \cdot 72$ & $\infty$ & $*$ \\
Pisa & & & \\
$* K^{\mathrm{F}}=\rho^{\mathrm{F}}\left(V_{\mathrm{P}}^{\mathrm{F}}\right)^{2}$ with $V_{\mathrm{P}}^{\mathrm{F}}=V_{\mathrm{P}}^{\mathrm{W}}(T)$ considered to be a function of temperature only,
\end{tabular}

according to Marczak (1997). In this study it was assumed that $T=10^{\circ} \mathrm{C}$ at both sites.

0.27. At the Sicily foundation site (see Fig. 14) the predicted values of porosity range from 0.26 to 0.34 for depths between $22 \mathrm{~m}$ and $70 \mathrm{~m}$ and from 0.09 to about 0.29 at greater depths. In Fig. 14 it is interesting to note that the decrease in porosity at depths greater than about $70 \mathrm{~m}$ coincides with the change in age of the geologic formation from Holocene to Pleistocene.

As mentioned in the previous section, the soil deposits at the Messina Strait are coarse-textured at both sites, and as a result no undisturbed specimens were retrieved from the boreholes. For this reason, no independent measurement of porosity is available to compare with the predicted values of $n$ plotted in Figs 13 and 14.

This comparison of the predicted values of porosity with independent laboratory measurements was instead possible at the Pisa site, where the subsoil conditions also include fine- textured soils such as silts and clays. Fig. 15 shows on the same chart the values of $n$ predicted by equation (13) and those determined in the laboratory on the retrieved undisturbed specimens (Costanzo, 1994). The comparison between the measured and predicted values of porosity is excellent, particularly at depths less than $30 \mathrm{~m}$. At greater depths there are only three values of porosity measured in the laboratory, at $31.5,33.4$ and $35.8 \mathrm{~m}$ respectively, which tend to be underestimated by the predicted values of $n$. From Fig. 15 it can also be noted that, similar to what happened at the Messina Strait sites, at Pisa the values of porosity computed from equation (13) also show, in general, a weak dependence on the unknown parameter $v^{\mathrm{SK}}$.

For some of the time histories recorded at Pisa during cross-hole measurements, Fourier spectra were computed to determine the frequency content of the stress waves generated 


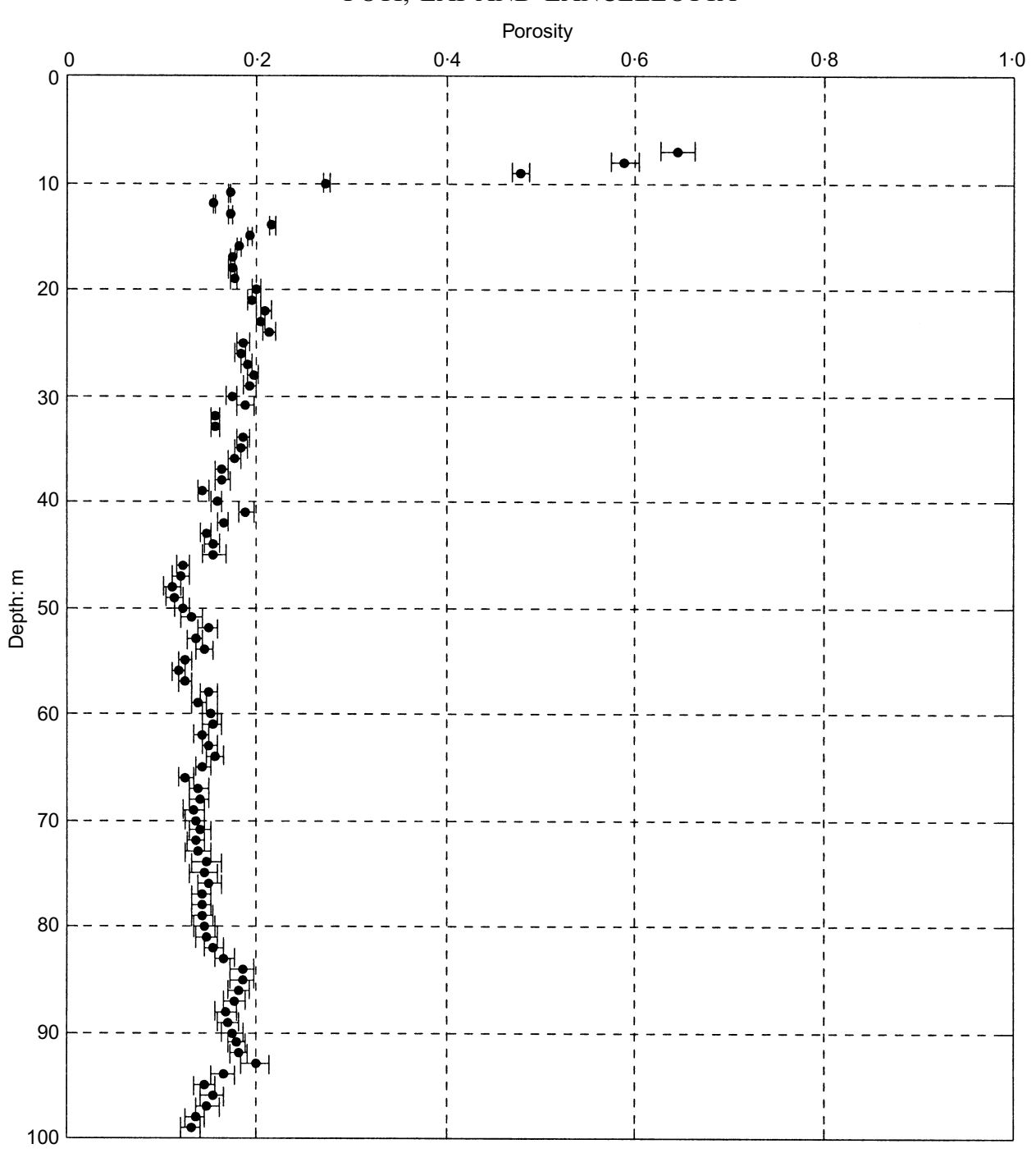

Fig. 13. Porosity predicted at Messina Strait, Calabria foundation site, using Biot's theory and measured $V_{\mathrm{P}}, V_{\mathrm{S}}$

in the porous medium, particularly in relation to the value of the characteristic frequency $f_{\mathrm{c}}=\omega_{\mathrm{c}} / 2 \pi$. Figs 16 and 17 show the time histories and Fourier spectra of the signals at $20 \mathrm{~m}$ and $60 \mathrm{~m}$ depth respectively. Fig. 16 refers to a measurement performed by using a piezoelectric source specifically designed to generate an impulse rich in $\mathrm{P}$-wave energy (SOLGEO S.r.1, 2001, private communication). From the figure it can be seen that most of the frequencies of the Fourier spectrum fall in the range $100-2000 \mathrm{~Hz}$ and, for the soil texture encountered in the Pisa formations (see Fig. 12), are far below the characteristic frequency, $f_{\mathrm{c}}$ (see Table 1). Fig. 17 shows the time history and Fourier spectrum of the signal measured at $60 \mathrm{~m}$ depth using a source specifically designed for the excitation of S-waves. In this case most of the energy of the spectrum falls in the range $200-400 \mathrm{~Hz}$.

\section{CONCLUDING REMARKS}

This paper has shown how the theory of linear poroelastodynamics in the low-frequency limit (that is assuming that no relative movement occurs between the fluid and the solid phase) developed by Biot (1956a; 1956b) can profitably be used for determining porosity in fluid-saturated porous media from measured shear and compression wave velocities. Whereas in fine-textured soils such as silts and clays poros- ity can be easily measured in the laboratory on undisturbed specimens, in coarse-textured materials undisturbed sampling is difficult, and the procedure illustrated in this article represents a simple but effective way to estimate the porosity in situ.

A particular aspect of the procedure is that its application requires only measurement of $V_{\mathrm{P}}$ and $V_{\mathrm{S}}$ of the medium, which are quantities that are routinely measured in geotechnical engineering using seismic prospecting methods such as cross-hole and down-hole tests. The other parameters required for the implementation of the method are four physical constants, which assume fairly standard numerical values, and the Poisson ratio of the (evacuated) soil skeleton. The latter parameter is a priori unknown, but numerical simulations and experimental data have shown that the computed values of porosity are not very sensitive to $v^{\mathrm{SK}}$ for the whole range of variability of this parameter.

The method has been successfully applied to estimate porosity at two sites in Italy where cross-hole seismic data were available. In one of these two sites (Pisa) independent values of porosity measured in the laboratory on undisturbed specimens were also available for comparison. The results show very good agreement between the values of porosity predicted by the theory and those measured in the laboratory. 


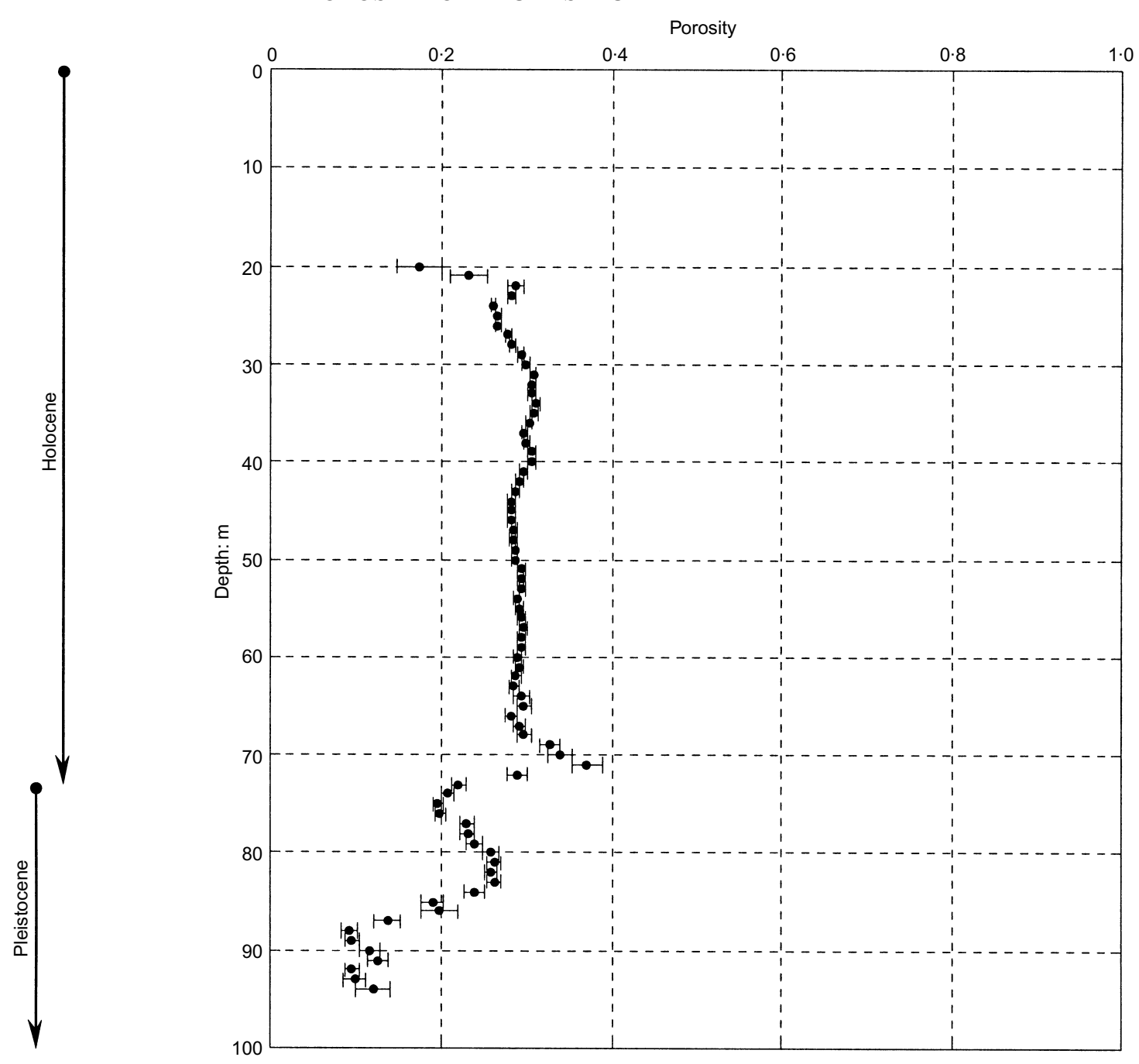

Fig. 14. Porosity predicted at Messina Strait, Sicily foundation site, using Biot's theory and measured $V_{\mathrm{P}}, V_{\mathrm{S}}$

\section{ACKNOWLEDGEMENTS}

The authors would like to thank Studio Geotecnico Italiano S.r.l. and particularly Professor Jamiolkowski for permission to use the seismic cross-hole data at both sites of Pisa and Messina Strait, and for the support given to the second author in performing the numerical simulations and editing the manuscript. Also, Professor Jamiolkowski's valuable suggestions in reviewing the manuscript are greatly acknowledged. A special word of appreciation goes to Dr Fioravante and $\mathrm{Mr}$ Signori of SOLGEO S.r.l. for providing the time histories of the cross-hole measurements conducted at the Pisa site, and to Dr Costanzo of Politecnico di Torino for making available the laboratory data on the porosity of the Pisa formations. Finally, the authors would like to express a sincere note of gratitude to Professor G. Rix of the Georgia Institute of Technology (Atlanta) and to Professor K. Wilmanski of the Weierstrass Institute for Applied Analysis and Stochastics (Berlin) for their valuable suggestions and comments in reviewing the manuscript.

\section{NOTATION}

$C$ Biot's constant (defined by equation (2b))

$D$ Biot's constant (defined by equation (2d))

$e_{\min }$ minimum void ratio

$e_{\max }$ maximum void ratio $f_{\mathrm{c}}$ characteristic frequency $\left(f_{\mathrm{c}}=\omega_{\mathrm{c}} / 2 \pi\right)$

$g$ acceleration of gravity $\left(g=9.80665 \mathrm{~m} / \mathrm{s}^{2}\right)$

$G_{\max }$ initial shear modulus

$G$ shear modulus of soil skeleton

$G^{\mathrm{SK}}$ shear modulus of evacuated soil skeleton

$H$ Biot's constant (defined by equation (2a))

$k$ coefficient of permeability of porous medium

$K^{\mathrm{S}}$ bulk modulus of soil particles

$K^{\mathrm{F}}$ bulk modulus of pore fluid

$K^{\mathrm{SK}}$ bulk modulus of soil skeleton

$M$ Biot's constant (defined by equation (2c))

$n$ porosity

$p^{\mathrm{F}} \quad$ pressure of pore fluid

$\hat{p}^{\alpha}$ momentum supply vector of the $\alpha$ th component $(\boldsymbol{\alpha}=\mathrm{S}, \mathrm{F})$

$t$ time

$\mathbf{T}^{\mathrm{S}}$ partial stress tensor of soil skeleton

$\mathbf{T}^{\mathrm{F}}$ partial stress tensor of pore fluid

$\mathbf{u}^{\mathrm{S}}$ displacement vector of soil skeleton

$\mathbf{u}^{\mathrm{F}} \quad$ displacement vector of pore fluid

$V^{\mathrm{P}}$ dilatational wave velocity of the first kind (P1wave)

$V_{\mathrm{P}}^{\mathrm{SK}}$ dilatational wave velocity of the evacuated soil skeleton

$V_{\mathrm{P}}^{\mathrm{F}}$ velocity of propagation of sound in water $\left(V_{\mathrm{P}}^{\mathrm{F}}=V_{\mathrm{P}}^{\mathrm{W}}\right)$

$V_{\mathrm{S}}$ rotational or shear wave velocity (S-wave) 


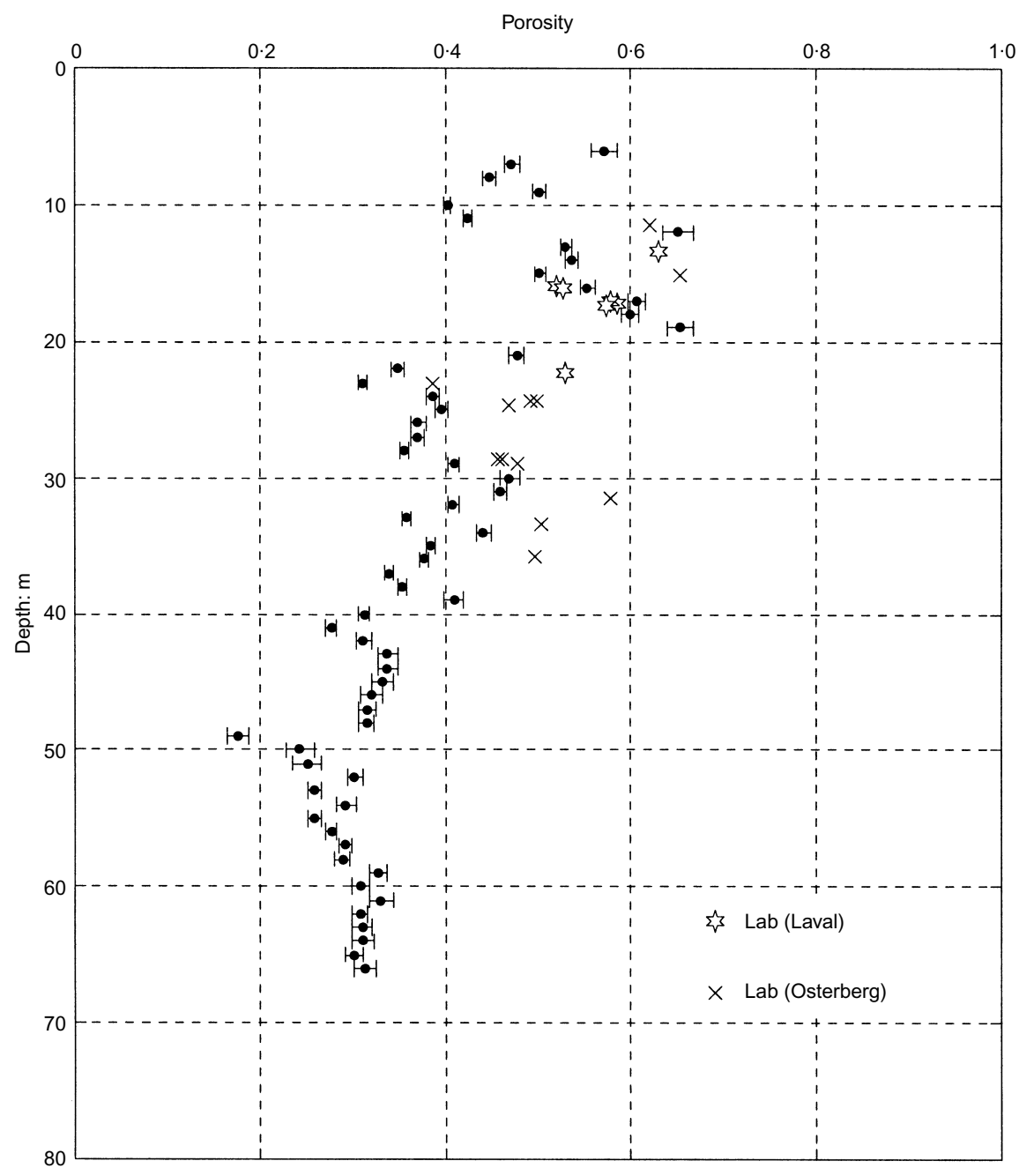

Fig. 15. Porosity predicted at Pisa site using Biot's theory and measured $V_{\mathrm{P}}, V_{\mathrm{S}}$ : comparison with independent data from laboratory (Costanzo, 1994)
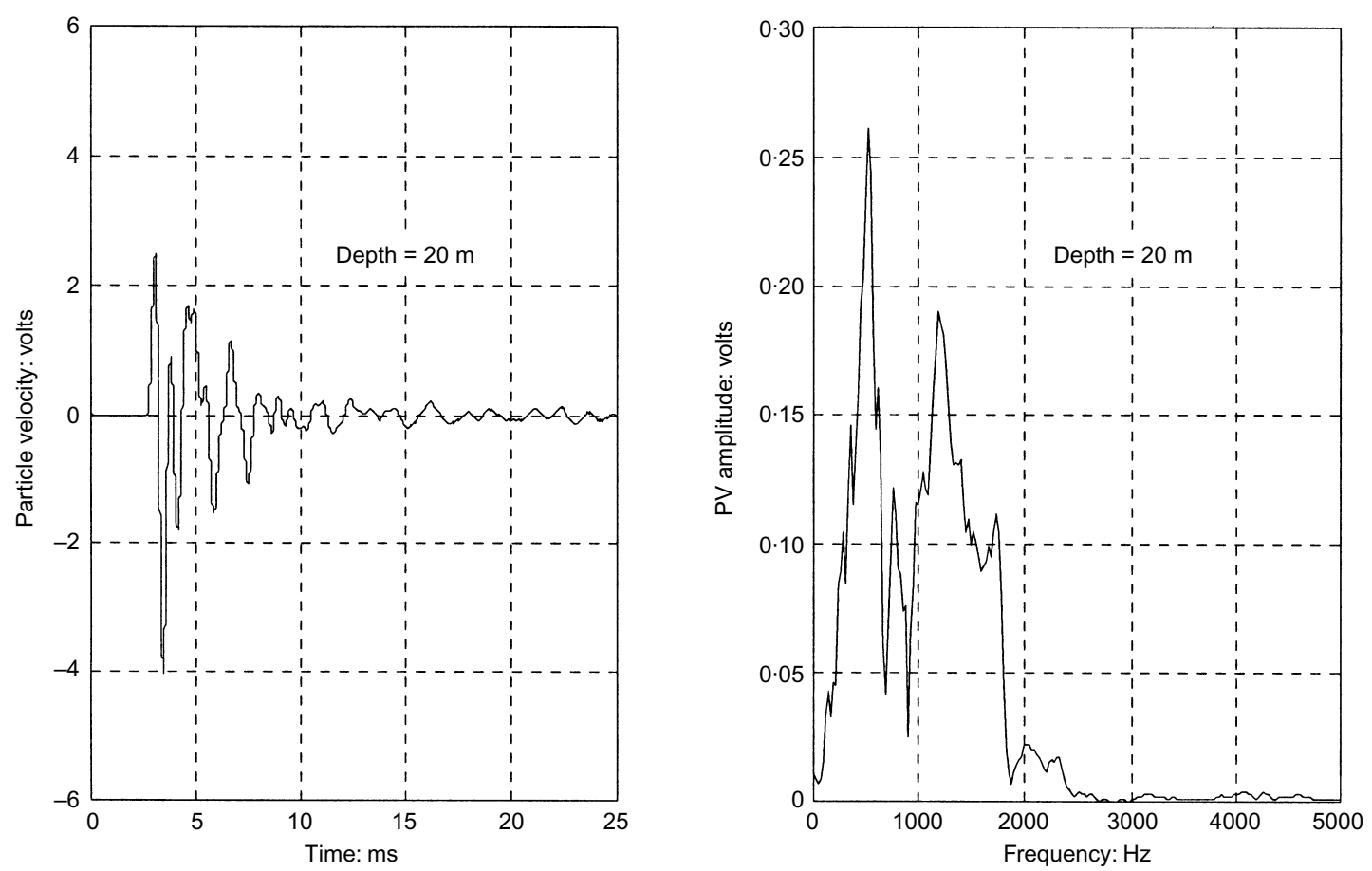

Fig. 16. Time histories and Fourier spectra of signals recorded at Pisa during cross-hole seismic testing: measurements using a P-wave source 

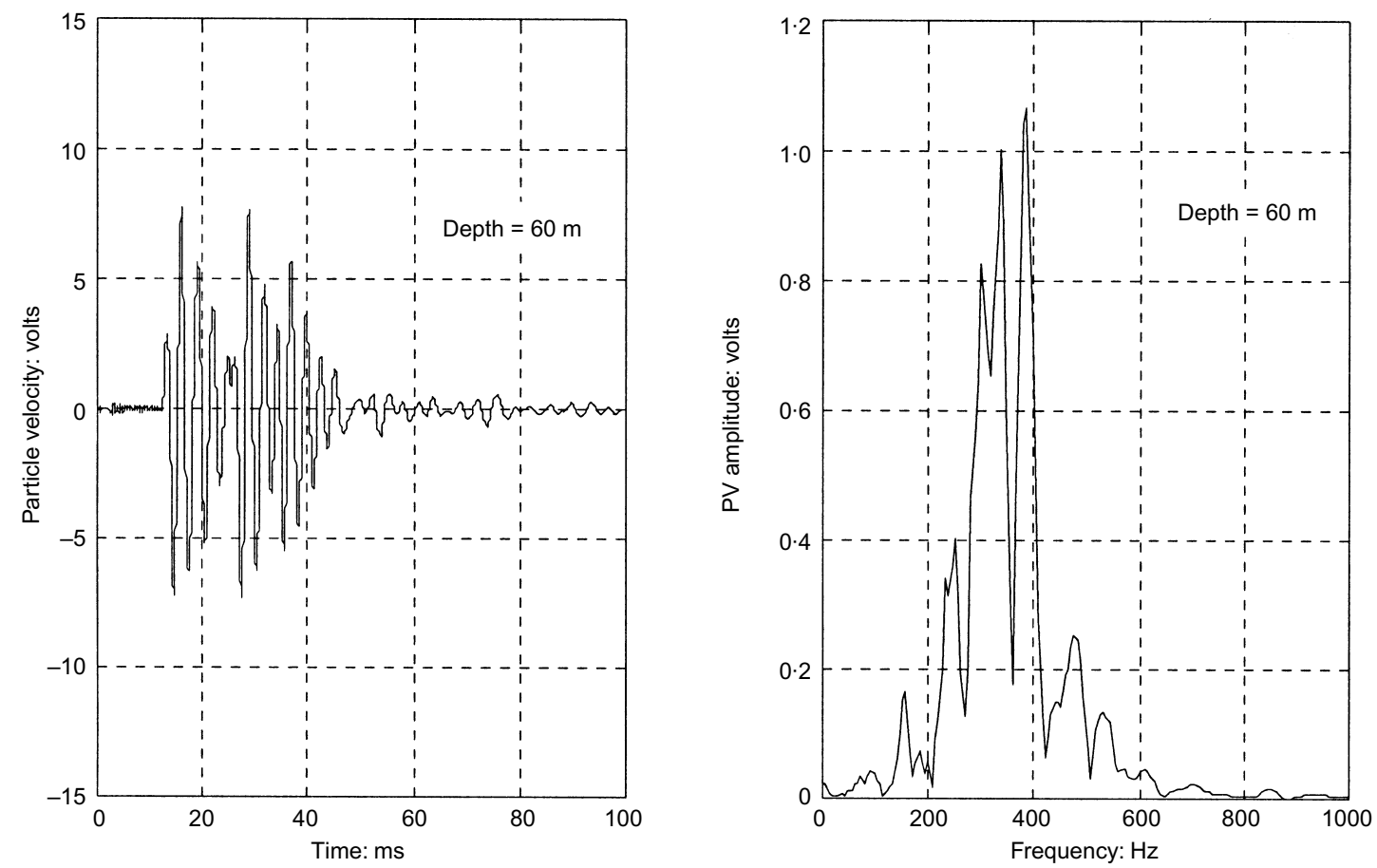

Fig. 17. Time histories and Fourier spectra of signals recorded at Pisa during cross-hole seismic testing: measurements using a S-wave source

$V_{\mathrm{S}}^{\mathrm{SK}}$ rotational or shear wave velocity of the evacuated soil skeleton

$v^{\mathrm{SK}}$ Poisson ratio of the evacuated soil skeleton

$\rho$ mass density of porous medium

$\rho_{\mathrm{S}}$ mass density of soil particles

$\rho^{\mathrm{SK}}$ dry mass density of soil skeleton

$\rho^{\mathrm{F}} \quad$ mass density of pore fluid

$\rho^{\mathrm{a}}$ apparent additional mass density

$\omega_{\mathrm{c}}$ characteristic circular frequency (defined by equation (7))

$\nabla^{2} \quad$ Laplacian operator

1 identity [3 $\times 3]$ matrix

$\mathscr{F}\{\ldots\}$ functional relationship $\mathrm{R}^{8} \rightarrow \mathrm{R}$

$[\ldots]^{\mathrm{T}}$ transpose operator

\section{REFERENCES}

Bates, C. R. (1989). Dynamic soil property measurements during triaxial testing. Géotechnique 39, No. 4, 721-726.

Belogol'skii, V. A., Sekoyan, S. S., Samorukova, L. M., Stefanov, S. R. \& Levtsov, V. I. (1999). Pressure dependence of the sound velocity in distilled water. Measurement Tech. 42, No. 4, 406-413.

Biot, M. A. (1956a). Theory of propagation of elastic waves in a fluid-saturated porous solid. I. Lower frequency range. J. Acoust. Soc. Am. 28, 168-178.

Biot, M. A. (1956b). Theory of propagation of elastic waves in a fluid-saturated porous solid. II. Higher frequency range. J. Acoust. Soc. Am. 28, 179-191.

Bourbie, T., Coussy, O. \& Zinszner, B. (1987). Acoustics of porous media. Paris: Editions Technip.

Bowen, R. M. (1982). Compressible porous media models by use of the theory of mixtures. Int. J. Engng Sci. 20, No. 6, 697-735.

Carmichael, R. S. (1989). Practical handbook of physical properties of rocks and minerals. Florida, USA, Boca Raton: CRC Press.

Costanzo, D. (1994). Prove meccaniche sull'argilla di Pisa. PhD dissertation, Politecnico di Torino, Italy (in Italian).

Costanzo, D., Jamiolkowski, M., Lancellotta, R. \& Pepe M. C. (1995). Leaning Tower of Pisa: description of the behaviour. Proc. 11th African Reg. Conf. Soil Mech. Found. Engng, Cairo, 1, 1-55.

Crova, R., Jamiolkowski, M., Lancellotta R. \& Lo Presti, D. C. F.
(1992). Geotechnical characterization of gravelly soils of Messina Strait site. Proceedings Wroth Memorial Symposium, 1, 199-218, Oxford.

Coussy, O. (1995). Mechanics of porous continua, p. 455. New York, John Wiley \& Sons.

Dyvik, R. \& Madshus, C. (1985). Lab measurements of $G_{\max }$ using bender elements. In Advances in the art of testing soils under cyclic conditions (ed. V. Khosla), pp. 186-196. New York: ASCE.

Gajo, A. (1996). The effects of inertial coupling in the interpretation of dynamic soil tests. Géotechnique 46, No. 2, 245-257.

Hofman, B. A., Sego, D. C. \& Robertson, P. K. (2000). In situ ground freezing to obtain undisturbed samples of loose sand. Journal of Geotechnical and Geoenvironmental Engineering, 126, No. 11, 979-989.

Kramer, S. L. (1996). Geotechnical earthquake engineering, p. 653. Englewood Cliffs, NJ: Prentice Hall.

Lancellotta, R. (2001). Coupling between the evolution of a deformable porous medium and the motion of the fluids in the connected porosity. In Springer Volume on Porous Media to honour R. de Boer (eds W. Ehlers and J. Bluhm), preprint. Berlin: Springer-Verlag.

Lings, M. L. \& Greening, P. D. (2001). A novel bender/extender element for soil testing. Géotechnique 51, No. 8, 713-717.

Marczak, W. (1997). Water as a standard in the measurements of speed of sound in liquids. J. Acoust. Soc. Am. 102, No. 5, $2776-2779$.

Miura, K., Yoshida, N. \& Kim, Y. S. (2001). Frequency dependent property of waves in saturated soil. Soils Found. 41, No. 2, 1-19.

Salem, H. S. (2000). Poisson's ratio and the porosity of surface soils and shallow sediments, determined from seismic compressional and shear wave velocities. Géotechnique 50, No. 4, 461-463.

Stoll, R. D. (1989). Sediment acoustics: Lecture notes in earth sciences. Berlin: Springer-Verlag.

Stretto di Messina S.p.A. (1989). Indagine geotecnica per acquisire le informazioni sulla natura del terreno e sulle sue caratteristiche atte alla progettazione delle fondazioni del Ponte sullo Stretto di Messina. Soluzione ad una luce. Zona fondazione (in Italian).

Wilmanski, K. (1998). Thermomechanics of continua. Berlin: Springer-Verlag.

Wilmanski, K. (2001). Some questions on material objectivity arising in models of porous materials. In Rational continua, classical and new: A collection of papers dedicated to G. Capriz (ed. M. Brocato, Milan, Italy), Springer, 149-161. 